\title{
Passivity Deformation Approach for the Thermodynamics of Isolated Quantum Setups
}

\author{
Raam Uzdin ${ }^{1, *}$ and Saar Rahav ${ }^{2, \dagger}$ \\ ${ }^{1}$ Fritz Haber Research Center for Molecular Dynamics, Hebrew University of Jerusalem, Jerusalem 9190401 , \\ Israel \\ ${ }^{2}$ Schulich Faculty of Chemistry, Technion-Israel Institute of Technology, Haifa 3200008, Israel
}

(Received 5 March 2020; revised 4 September 2020; accepted 26 January 2021; published 2 March 2021)

Recently implemented quantum devices such as quantum processors and quantum simulators combine highly complicated quantum dynamics with high-resolution measurements. We present a passivity deformation methodology that sets constraints on the evolution of such quantum devices. The approach yields bounds that are often tighter, and thus more predictive, than the quantum microscopic analogue of the second law of thermodynamics. In particular, (i) it yields tight bounds even when the environment is microscopic; (ii) it successfully handles the ultracold limit; (iii) it enables one to account for constrained dynamics; and (iv) it bounds observables that do not appear in the second law of thermodynamics. Furthermore, this framework provides insights into nonthermal environments, correlated environments, coarse graining in microscopic setups, and the ability to detect heat leaks. Our findings can be explored and used in physical setups such as trapped ions, superconducting circuits, neutral atoms in optical lattices, and more.

DOI: 10.1103/PRXQuantum.2.010336

\section{INTRODUCTION}

The theory of thermodynamics emerged during the industrial revolution. This celebrated theory was developed due to the pressing need to know how much coal a steam engine requires to accomplish a task. One of the strengths of the theory is its ability to make predictions that do not depend on the precise details of a specific engine. Instead, it provides universal laws (bounds) that apply to all systems and processes.

Rapid technological advances facilitate unprecedented ability to control and manipulate setups with highly pronounced quantum dynamics. Examples include dozens of interacting (atomic) spins in ion traps, neutral atoms in optical lattices, superconducting circuits, Rydberg atom lattices, etc. These setups are candidates for the realization of quantum technologies such as computation, simulations, communication, and more. Such applications require the ability to measure very specific observables that would, for instance, correspond to the result of quantum computation.

\footnotetext{
*raam@mail.huji.ac.il

†rahavs@technion.ac.il
}

Published by the American Physical Society under the terms of the Creative Commons Attribution 4.0 International license. Further distribution of this work must maintain attribution to the author(s) and the published article's title, journal citation, and DOI.
For example, in ion traps or superconducting circuits, it is possible to measure quantities such as the polarization of specific spins, their mutual polarization covariance (correlation), or the population of some preferred states. We refer to such observables as "fine grained" to distinguish them from observables that characterize the whole system, such as energy, volume, entropy, etc.

Since fine-grained experimental data are presently available, it is desirable to have a theory that can make predictions on this type of quantities. One possible approach is to model all the details of the setup at hand and solve or simulate the process of interest. Such an approach has to be repeated if the setup is driven using a different protocol or initialized in a different initial condition. Furthermore, this method is not feasible in quantum computations and simulators that attempt to solve problems that are computationally hard (classically). A different approach, more in the spirit of thermodynamics, is to identify constraints that are applicable to a whole class of processes without the need to explicitly solve for the evolution. This is the approach we take in the present paper.

The utility of thermodynamiclike bounds on finegrained quantities is illustrated using the examples depicted in Fig. 1. In Fig. 1(a) we illustrate the use of thermodynamiclike inequalities for detecting undesired coupling of a quantum system to some (possibly unknown) environment. Modern quantum devices such as quantum computers and simulators require a high level of isolation 
(a)

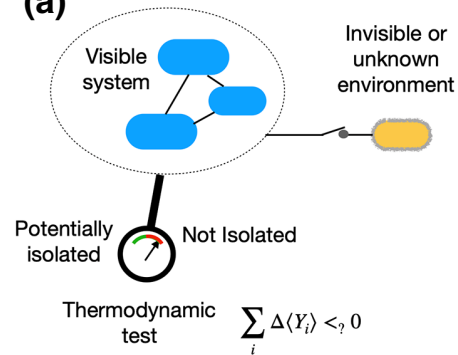

(b)

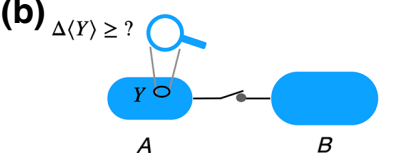

(c)

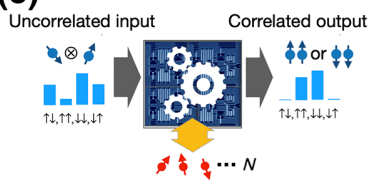

FIG. 1. Inequalities as a tool to study and describe the evolution of quantum machines and devices. (a) Violation of inequalities can be used to deduce coupling to an environment that is assumed to be negligible. (b) The inequalities can reveal by how much one can vary the expectation value of an observable by coupling a system to another small system. (c) Setting the limitations on the operation of exotic heat engines, namely machines that use thermal resources to try and change the value of a fine-grained observable (see the description of the machine in the main text).

from the rest of the world. The processor constitutes the visible system in which we can measure and control its initial state. Let us assume that the processor runs a complicated task that cannot be simulated classically. Is it possible to detect a coupling of the visible system to some environment without knowing the circuit (algorithm) or the details of the environment? The methods presented in this paper allow us to derive several inequalities that should hold as long as the assumption that the setup is isolated is justified. If an experiment shows that one of these inequalities is violated, it indicates that the system is not isolated. Our approach allows us to obtain inequalities that are tighter than the microscopic version of the second law and therefore serve as better detectors for heat leaks. It is interesting to point out that the second law cannot be saturated when the sum of local entropies increases. Nevertheless the inequalities we find can be saturated even when the sum of local entropies increases. See Ref. [1] for an experimental demonstration of heat leak detection based on the global passivity framework introduced in Ref. [2]. The approach is in the same spirit as Ref. [3] where violations of the fluctuation relation (an equality) were used to identify errors in a quantum annealer.

In Fig. 1(b) we depict a generic scenario of interest. From a fundamental perspective, it is imperative to understand what are the limitations on the manipulation of some observable in system 1 by interacting with system 2. The second law is an example of such a limitation where the observables are related to energies of the subsystems. In the microscopic world, it is possible to measure and manipulate many different quantities, and it is unclear what limits the changes of these observables. In this paper, we address this general question. Finally, in Fig. 1(c) we show an example of an "exotic heat machine" [4],

which is a more concrete realization of the general scheme presented in Fig. 1(b). Exotic heat machines use thermodynamic resources (work, heat, thermal environments) to execute a nonthermodynamic task (i.e., a task different from work extraction, cooling, etc.). Such machines were used in Refs. [5,6] to create steady-state entanglement. In the example in Fig. 1(c), the machine uses an $N$-spin thermal environment to increase the correlation between two initially uncorrelated spins. In particular, the goal is to increase the probability that spins 1 and 2 are aligned. The system observable that represents the alignment is $A=$ $\left|0_{1} 0_{2}\right\rangle\left\langle 0_{1} 0_{2}|+| 1_{1} 1_{2}\right\rangle\left\langle 1_{1} 1_{2}\right|$. A more quantum fine-grained task would be to increase the expectation value of some entanglement witness. Since $\langle A\rangle$ is not the energy of a subsystem, the second law of thermodynamics is not suitable for constraining the changes of $\langle A\rangle$. In particular, it is interesting to understand how much heat and work are needed for changing $\langle A\rangle$ and how the performance depends on the size of the small environment and its initial temperature. In this paper, we present a framework that can generate bounds that address some of these questions.

Our approach, passivity deformation, uses the recently introduced notion of global passivity [2] as a starting point, yet it quickly deviates from global passivity in order to overcome some of its inherent limitations. Global passivity produces a family of inequalities on observables, one of which is the second law. The predictive power of global passivity bounds was experimentally demonstrated using the IBM superconducting quantum processors [1]. By checking the validity of these inequalities, it was possible to detect heat leaks that the second law and other thermodynamic frameworks could not detect. In Ref. [2] they were also used to detect subtle Maxwell demons (i.e., weak feedback operations) in numerical simulations.

Despite being based on a different mathematical framework, global passivity still shares the same limitations of the second law when it is applied to small quantum setups. By "second law" we refer to Eq. (11), which is the analogue of the Clausius inequality in microscopic quantum setups. Although this form of the second law can be rigorously derived, it is often very loose in microscopic setups, which severely limits its predictive power. The first limitation, as discussed earlier, is the inability to restrict the changes of the expectation value of a fine-grained quantity. This however could be viewed as a feature and not as a limitation since Eq. (11) does not aim to deal with such an observable. We refer the reader to Refs. [7-10] for an entropic approach (i.e., not observable based) to finegrained features. The second limitation is the "ultracold catastrophe," in which one considers processes involving a subsystem that is initially at zero temperature. There the term $Q_{c} / T_{c}$ dominates the inequality of the second law, and the only possible prediction is that $Q_{c} \leq 0\left(Q_{c}<0\right.$ refers to heat leaving the system, later we use $q_{c}>0$ to describe energy entering the bath). However, when $T_{c}$ is 
low enough, the environment effectively starts in its ground state, so clearly the average energy cannot go further down. Thus, in this case, the second law does not add any new information. See Refs. [11-13] for additional interesting approaches for handling the ultracold catastrophe. The third limitation is that the second law and the global passivity bounds are unattainable when the environments go out of equilibrium. This is typically the case in microscopic setups where the heat capacities are very small. This unattainability tells us that the process is inherently irreversible. However, obtaining a tighter bound that is also attainable will clearly enhance our ability to predict what is achievable in such processes. Finally, the fourth limitation is the difficulty in taking into account constraints that limit the dynamics to a restricted set of unitaries (global unitaries that include all the elements of interest). This typically happens when the interaction that drives the dynamics can be divided into disconnected sets of levels (orthogonal Hilbert spaces). That is, the interaction takes place between the states in the set $A$, and/or between the states in set $B$, but not between a state in $A$ and a state in $B$. A simple example is energy conserving interactions where the sets are the total energy shells of the system. Such disconnected sets are always associated with a conservation law since the probability to be in each set is conserved by the constrained interaction.

Once the dynamics is constrained, it is often the case that the dynamics saturating some unconstrained bound cannot be implemented. As a result, this bound is looser than required and the predictions it makes on the observed quantities of interest are less informative. We aim to provide tools that take into account the fact that the evolution takes place in disconnected sets of levels, and produce bounds that can be saturated despite the constraint. This can be used, for example, for detecting weaker heat leaks. Notably, the passivity deformation framework simultaneously solves these four limitations. However, we do not claim that the resulting inequalities are unique or optimal, but we demonstrate they produce tighter bounds compared to Eq. (11), and other recently derived variations of the second law in the microscopic setup. In a companion paper to the present one [1], we have utilized the IBM platform mentioned above to experimentally demonstrate that in the task of heat leak detection, passivity deformation can outperform both the second law and the global passivity inequalities. This demonstration provides a positive indication that this framework is relevant for modern experiments and that it increases the predictive power of thermodynamiclike inequalities in such setups.

Recent years have seen important developments in our understanding of the thermodynamics of small quantum systems. Stochastic thermodynamics allows one to assign thermodynamic quantities such as heat or work to a single trajectory of particles (e.g., a colloidal particle or a molecular motor $[14,15])$. Quantum thermodynamics aims to identify the thermodynamic role of purely quantum effects such as coherence, measurement back action, or entanglement [16-19]. Despite their success, both approaches are not well suited for the goals discussed above due to their focus on the energy (work, heat, and their fluctuations) as the observable of interest. A relevant approach to microscopic thermodynamics was recently suggested by Strasberg and Winter $[9,10]$. Their approach is based on the notion of observational entropy $[7,8]$. They demonstrated that an observational entropy of any measurement in the system and energy in an environment satisfies a second-law-like inequality. As a result, one can obtain inequalities that constrain fine-grained quantities. A crucial difference between the results of Refs. [9,10] and the approach presented here is that the inequalities we derive involve changes in expectation values. In contrast, the second law of Refs. $[9,10]$ involves changes in entropies, namely quantities of the form $-\sum_{i} p_{i} \ln p_{i}$, which are harder to measure.

Our paper is structured as follows. After reviewing the notions of passive operators and global passivity in Secs. II, in III we describe the essence of the passivity deformation method. In addition to studying the tightness of the new bounds and their physical meaning, we also show that constraints on the dynamics that are associated with conservation laws can be integrated and yield even better bounds. The section ends with several illustrative examples. In Sec. IV we introduce an intuitive graphical representation of our framework, which is exploited for deriving several useful bounds and insights without doing explicit calculations. For example, we obtain a more refined bound on information erasure compared to the Landauer bound. We then use our framework to address the ultracold catastrophe. At the end of this section, we show that some nonthermal and potentially correlated environments can be treated on the same footing as thermal uncorrelated environments, where the deviation from thermal initial conditions reduces to using new effective temperatures in the familiar second law. In Sec. V passivity deformation is utilized to study coarse graining within the framework of passivity, and also to show that some heat leaks cannot be detected with passivity-based constraints. We conclude in Sec. VI.

\section{PASSIVE STATES, PASSIVE OPERATORS, AND GLOBAL PASSIVITY}

\section{A. Passive states}

Passive states (passive density matrices) were introduced for studying how much work can be extracted from an isolated system by using external forces [20-25]. Mathematically, this requires finding the unitary transformation that brings the system to the lowest energy. Crucially, the notion of passivity is not limited to studies of energy changes. It can be applied to other observables as well. 
In Ref. [22] passivity was used for deriving a simplified form of the second law and later, independently, the potential of passivity for generating additional constraints on the dynamics of observables was studied in Ref. [2]. Consider a Hermitian operator $A$, and a system whose initial state is described by a density matrix $\rho_{0}$. The operator $A$ may be the Hamiltonian of the whole system, a subsystem, or may also describe other observables, such as angular momentum, or projection operators onto specific subspaces. One can then ask what is the minimal value of $\langle A\rangle=\operatorname{tr}(\rho A)$ that is reachable from the initial state by a unitary transformation. The state obtained by this optimal unitary is called a "passive state" $\rho_{A \text { pass }}$ (with respect to the operator $A$ ). By construction, one can write the inequality

$$
\langle A\rangle \geq \min _{\text {all } \mathrm{U}} \operatorname{tr}\left(U \rho U^{\dagger} A\right)=\operatorname{tr}\left(\rho_{A \text { pass }} A\right),
$$

which holds for all unitaries $U$. The passive state $\rho_{A \text { pass }}$ has an explicit expression. The operator $A$ can be written in terms of its eigenvalues and eigenvectors, $A=$ $\sum_{i} a_{i}\left|a_{i}\right\rangle\left\langle a_{i}\right|$, with $a_{i+1} \geq a_{i}$. A general initial density matrix has the form $\rho_{0}=\sum_{i} r_{i}\left|r_{i}\right\rangle\left\langle r_{i}\right|$. A density matrix that is passive with respect to the operator $A$ will then have the form

$$
\rho_{A \text { pass }}=\sum_{i} r_{i}\left|a_{i}\right\rangle\left\langle a_{i}\right|
$$

with $r_{i+1} \leq r_{i}$. Thus, the optimal unitary is simply $U_{\text {opt }}=$ $\sum_{i}\left|a_{i}\right\rangle\left\langle r_{i}\right|$. The ordering of $r_{i}$ with respect $a_{i}$ is crucial for passivity (see the proof in Ref. [23]). The conditions for passivity given in Eq. (2) can also be written as

$$
\left[A, \rho_{A \text { pass }}\right]=0, \quad\left\langle a_{i+1}\left|\rho_{A \text { pass }}\right| a_{i+1}\right\rangle \leq\left\langle a_{i}\left|\rho_{A \text { pass }}\right| a_{i}\right\rangle .
$$

In particular, it can be used for the evolution operator of a setup that includes all the elements that interact with each other. Thus, if a setup was prepared in an initial state that is passive with respect to an operator $A$, i.e., it already has the minimal value of $\langle A\rangle$, then any subsequent unitary evolution must satisfy the inequality

$$
\Delta\langle A\rangle=\operatorname{tr}\left(\rho_{f} A\right)-\operatorname{tr}\left(\rho_{0} A\right) \geq 0
$$

for $\rho_{0}=\rho_{A \text { pass. }}$. Moreover, by linearity, this inequality also holds if the evolution is described by a mixture of unitaries

$$
\rho_{f}=\sum_{k} p_{k} U_{k} \rho_{0} U_{k}^{\dagger} .
$$

This map is unital, i.e., a fully mixed state (the identity operator plus trace normalization) is a fixed point of the map. Simply put, Eqs. (4) and (5) state that, starting with the minimal value obtained by unitaries, the expectation value can only grow with respect to its initial value.

\section{B. Passive operators}

Passivity is not a property of the density matrix alone but a relation between a density matrix and an observable (an operator). A given initial density matrix $\rho_{0}$ may be nonpassive with respect to the Hamiltonian, but passive with respect to other operators. In contrast to the previous section where passive states were defined, we now take the complementary point of view and discuss passive operators. Here, the initial density matrix is given and written in the basis of decreasing eigenvalues: $\rho=\sum r_{i}^{\prime}\left|r_{i}^{\prime}\right\rangle\left\langle r_{i}^{\prime}\right|$ with $r_{i+1}^{\prime} \leq r_{i}^{\prime}$. A passive operator $A_{\rho_{0} \text { pass }}$ with respect to $\rho_{0}$ satisfies

$$
\begin{aligned}
{\left[A_{\rho_{0} \text { pass }}, \rho_{0}\right] } & =0, \\
\left\langle r_{i+1}^{\prime}\left|A_{\rho_{0} \text { pass }}\right| r_{i+1}^{\prime}\right\rangle & \geq\left\langle r_{i}^{\prime}\left|A_{\rho_{0} \text { pass }}\right| r_{i}^{\prime}\right\rangle,
\end{aligned}
$$

for any $i$. Such operators satisfy

$$
\Delta\left\langle A_{\rho_{0} \text { pass }}\right\rangle \geq 0
$$

for any mixture of unitaries (5). This is illustrated in Fig. 2(a): when a setup undergoes various unitary evolutions (light green curves), the expectation value of a passive operator will never go below its initial value (red zone). In contrast, for a nonpassive operator (dark green curves), there is always a unitary that reduces the expectation value below its initial value (i.e., enters the red zone).

In Figs. 2(b) and 2(c) examples of passive and nonpassive operators are depicted. The $x$ axis corresponds to $\left|r_{i}^{\prime}\right\rangle$, i.e., eigenstates sorted in decreasing order of probabilities (eigenvalues of $\rho_{0}$ ), as indicated by the line-dot curves. Note that in this example states $\left|3^{\prime}\right\rangle$ and $\left|4^{\prime}\right\rangle$ are degenerate (dotted ellipse), so their relative order is arbitrary and their position can be switched. The $y$ axis corresponds to $\left\langle r_{i}^{\prime}|\mathcal{C}| r_{i}^{\prime}\right\rangle$ (Fig. 2b) and $\left\langle r_{i}^{\prime}|D| r_{i}^{\prime}\right\rangle$ [Fig. 2(c)]. After exploiting the freedom to reorder degenerate eigenstates of $\rho_{0}$, Eq. (6) is satisfied for any state $\left|r_{i}^{\prime}\right\rangle$ in Fig. 2(b). Hence, operator $\mathcal{C}$ is passive with respect to $\rho_{0}$. Operator $D$ shown in Fig. 2(c) is not passive with respect to $\rho_{0}$.
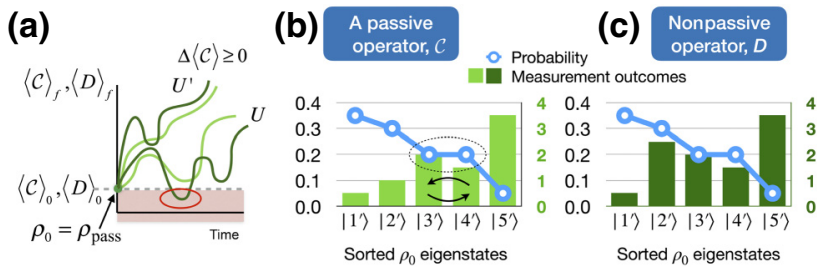

FIG. 2. (a) The expectation values of a passive operator $\mathcal{C}$ (light green) must increase under any unitary operation with respect to the initial value. The dark green curves show the expectation values of a nonpassive operator $D$. (b),(c) Graphical representations of passive and nonpassive operators in the basis of decreasing probabilities. 
The slight complication that arises due to degeneracies in $\rho_{0}$ or $A$ can be avoided by two means. One is to exploit the binary relation defined in Appendix A. The second option is to define the nonpassive operator $\bar{A}$ in the following way: given that $\left[\bar{A}, \rho_{0}\right]=0$ holds, if there are two eigenstates $i$ and $j$ for which $\left\langle i\left|\rho_{0}\right| i\right\rangle>\left\langle j\left|\rho_{0}\right| j\right\rangle$ and $\langle i|\bar{A}| i\rangle>\langle j|\bar{A}| j\rangle$ then $\bar{A}$ is nonpassive with respect to $\rho_{0}$. Any operator that commutes with $\rho_{0}$ and is not nonpassive is passive.

For a given initial state $\rho_{0}$, there are many operators that are passive with respect to it. Next, we show how to construct a passive operator with a clear physical interpretation.

\section{Global passivity}

In our previous work [2] we used $\rho_{0}$ itself to construct an operator that is passive with respect to it. There are many possible choices, yet one particular choice reveals a connection between passivity and the second law:

$$
\mathcal{B}=-\ln \rho_{0}^{\text {tot }} .
$$

Here, crucially, $\rho_{0}^{\text {tot }}$ is the density matrix of the whole setup, including both the system and the environments. Note that, by definition, the operator $\mathcal{B}$ does not change in time, i.e., $\langle\mathcal{B}\rangle_{t}=\operatorname{tr}\left[\rho^{\text {tot }}(t)\left(-\ln \rho_{0}^{\text {tot }}\right)\right]$. The passivity of $\mathcal{B}$ with respect to $\rho_{0}^{\text {tot }}$ immediately follows from $\rho_{0}^{\text {tot }}=e^{-\mathcal{B}}$. Consequently, it holds that

$$
\Delta\langle\mathcal{B}\rangle \geq 0
$$

for any mixture of unitaries. The quantum evolution of a microscopic system may not be unitary if it is not isolated from its environment. Nevertheless, the evolution of both the system and its nearby environments can be viewed as unitary if they are sufficiently isolated from the rest of the universe. In such cases, any process can be modeled as a unitary that acts on both the system and its local environment. The approach we present here makes this assumption. We use the term global passivity to highlight the fact that the processes we consider involve unitary evolution that acts on both the system and its local environments, in contrast to the standard notion of passivity where one subsystem is driven by an external field.

Without a meaningful physical interpretation, inequality (9) is just a mathematical result. Our previous work starts by pointing out a clear connection between global passivity and the second law. Let $\left\{\beta_{k}, H_{k}\right\}$ describe the inverse temperatures and Hamiltonians of a set of subsystems that act as environments. These potentially microscopic environments are termed "microbaths." In contrast to large baths, they may strongly deviate from thermal equilibrium when interacting with each other or with some external forces. Hence, their temperature refers only to their initial state. We consider a setup where several microbaths interact with each other (e.g., as in absorption refrigerators [26-32]). Such a setup can also describe heat engines and power refrigerators. Since $\rho_{0}=\exp \left(-\sum_{k} \beta_{k} H_{k}\right) / Z$ $\left[Z=\operatorname{tr}\left\{\exp \left(-\sum_{k} \beta_{k} H_{k}\right)\right\}\right.$ is a normalization factor], the global passivity of $\mathcal{B}$, Eq. (9) yields

$$
\sum_{k} \beta_{k} \Delta\left\langle H_{k}\right\rangle \geq 0
$$

which is the analogue of the second law for a set of potentially small quantum systems. For completeness, the analogue of the complete form of the classical Clausius inequality is given by [33-35]

$$
\Delta S_{\text {sys }}+\sum_{k} \beta_{k} \Delta\left\langle H_{k}\right\rangle \geq 0 .
$$

Here $\Delta S_{\text {sys }}$ is the change in the von Neumann entropy of the system. Our goal, however, is to obtain inequalities that constrain expectation values of observables. Quantities such as $\Delta S_{\text {sys }}$ are considerably harder to measure experimentally, so we wish to avoid their appearance in the inequalities when possible. In addition, Eq. (11) reduces to Eq. (10) under a periodic evolution of the system. While such periodic operation often takes place in steady state driven by large environments, it can also take place when the system acts as a catalyst for energy exchange between small environments (e.g., catalytic cooling and catalytic thermometry transformations [36]). Finally, the relation between Eqs. (11) and (10) was studied in Sec. IID of Ref. [2].

We emphasize once again that $\beta_{k}$ in Eqs. (10) and (11) refers only to the initial state of the environments. To identify $\Delta\left\langle H_{k}\right\rangle$ with the change in the average energy of the $k$ th microbath, it is essential that the Hamiltonian of the microbath at the end of the process is equal to the Hamiltonian at the beginning of the process. Furthermore, in Eqs. (10) and (11) the terms $\Delta\left\langle H_{k}\right\rangle$ are not automatically identified as "heat." Although one can do so, there are other legitimate alternatives (see Ref. [37] and Secs. 28.3.6 or III E of Ref. [4]). Ultimately, Eq. (10) refers to average energy changes in the microbaths and is independent of how heat and work are defined.

While Eqs. (10) and (11) resemble the familiar second law of thermodynamics, they deviate from the classical thermodynamics result in several important aspects: (i) the microbaths can be small, and may substantially deviate from their initial thermal state during the process; (ii) the dynamics may create entanglement and correlations between different subsystems; (iii) thermal relaxation with ideal heat baths are not included or assumed; and (iv) work can be done during the process, but some of it may be done on the microbaths and not only on the system of interest.

The example above shows both a systematic construction of a passive operator and a clear thermodynamic 
context. Yet, this example adds nothing new on top of the already known Eq. (10) [4,16,33-35,38]. The added value of the global passivity framework manifests when constructing additional globally passive operators. In particular, in Ref. [2] it was shown that $\mathcal{B}^{\alpha}$ is also passive with respect to $\rho_{0}^{\text {tot }}$ for any $\alpha>0$ [the more general form is $\operatorname{sgn}(\alpha) \mathcal{B}^{\alpha}$ for any real $\alpha$ ]. Crucially, in several cases we found that the resulting inequalities contain useful information that is not included in Eq. (10) or (11). As mentioned earlier, the added value of these inequalities has recently been experimentally demonstrated on the IBM quantum processor platform [1]. For the reader familiar with thermodynamic resource theory [39-43], we point out that the global passivity inequalities are different because they deal only with observable quantities.

As shown in Appendix A, global passivity can be formulated as a binary relation based on a matrix ordering function. The conditions for this binary relation to become an equivalence relation are discussed as well. While, for clarity, the paper is written in the conventional formalism of passivity, Eqs. (4) and (6), the formalism in Appendix A is highly useful when exploring consequences of passivity.

The global passivity approach described above is well suited for processes where a collection of quantum systems is prepared in a known initial density matrix and is then driven. The resulting inequalities tell us what cannot be achieved in any subsequent evolution. Yet, just like Eq. (11), global passivity posses the same limitations mentioned in the Introduction (briefly: lack of prediction for fine-grained observables, no attainability of bounds for a small environment, ultracold catastrophe, loose bound when the dynamics is constrained).

\section{THE PASSIVITY DEFORMATION APPROACH}

In what follows we present a new approach that overcomes the aforementioned limitations. Consider an observable of interest $A$ that satisfies $\left[A, \rho_{0}^{\text {tot }}\right]=0$. If $A$ is passive with respect to $\rho_{0}^{\text {tot }}$ then $\Delta\langle A\rangle \geq 0$, but if it is not, it is generally unclear what predication can be made on $\Delta\langle A\rangle$ without solving the full dynamics. In our approach, we construct a globally passive operator that contains $\Delta\langle A\rangle$ as well as other observables. The first step is to choose a passive operator, for instance, $\mathcal{B}=-\ln \rho_{0}^{\text {tot }}$, and use it to define the operator

$$
B(\xi)=\mathcal{B}+\xi A
$$

where $B(0)$ is globally passive by construction. It is expected that there is a finite range of $\xi$ values for which $B(\xi)$ is also globally passive and therefore satisfies $\Delta\langle B(\xi)\rangle \geq 0$. As explained later, for discrete and finite systems, there is always $\xi \neq 0$ for which $B(\xi)$ is globally passive.

Which values of $\xi$ should be used? Tighter and, therefore, more restrictive and informative inequalities for $\langle A\rangle$
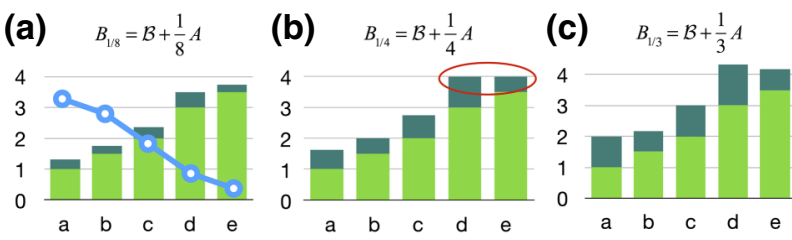

FIG. 3. Passivity deformation — when adding a small amount of a nonpassive operator (dark green) to a passive operator (light green), the combined operator created by the sum (depicted by the total height) may still be passive, as shown in (a). At some critical value, a new degeneracy forms (b). If the fraction of the nonpassive part is too large, passivity is lost (bar no longer monotonically increases) (c).

are obtained when $|\xi|$ is as large as possible. However, at some point, some of the eigenvalues of $B(\xi)$ become degenerate due to the change of $\xi$. Degeneracies inherited from $B(0)$ are irrelevant at this point —only those emerging from increasing $|\xi|$. At this critical value of $\xi$ the ordering of the operator changes and it stops being globally passive. This is illustrated in Fig. 3.

We denote by $\lambda_{k}^{(\mathcal{B})}\left(\lambda_{k+1}^{(\mathcal{B})} \geq \lambda_{k}^{(\mathcal{B})}\right)$ and $v_{k}^{(\mathcal{B})}$ the eigenvalues and eigenvectors of $\mathcal{B}$. Because of passivity, the probability of observing eigenstate $v_{k}^{(\mathcal{B})}$ decreases as $\lambda_{k}^{(\mathcal{B})}$ is increased. The notation $\lambda_{k}^{(A)} \stackrel{\circ}{=}\left\langle v_{k}^{(\mathcal{B})}|A| v_{k}^{(\mathcal{B})}\right\rangle$ refers to the eigenvalue of $A$ associated with eigenvector $v_{k}$ of $\mathcal{B}(A$ and $\mathcal{B}$ have the same eigenvectors since they commute). Note that, unlike $\lambda_{k}^{(\mathcal{B})}$, the $\lambda_{k}^{(A)}$ are not sorted in decreasing order. The condition for degeneracy between consecutive eigenvalues of $B(\xi)$ is $\lambda_{k+1}^{(\mathcal{B})}+\xi \lambda_{k+1}^{(A)}=\lambda_{k}^{(\mathcal{B})}+\xi \lambda_{k}^{(A)}$. Let us define $\xi_{k}=\left(\lambda_{k+1}^{(\mathcal{B})}-\lambda_{k}^{(\mathcal{B})}\right) /\left(\lambda_{k}^{(A)}-\lambda_{k+1}^{(A)}\right)$, where $k$ values that nullify the numerator or denominator are excluded since degeneracies in either $A$ or $\mathcal{B}$ do not affect the relative ordering of the two operators. Using $\xi_{k}$, we define

$$
\begin{gathered}
\xi_{+}=\min \left(\xi_{k}>0\right), \\
\xi_{-}=-\min \left(-\xi_{k}>0\right) .
\end{gathered}
$$

The operator $B(\xi)$ is then passive in the range $\xi_{-} \leq \xi \leq$ $\xi_{+}$. Since $\xi_{k}$ cannot take the value zero (due to the exclusion of $k$ values in the definition of $\xi_{k}$ ), there is a nontrivial $\xi$ for which $B(\xi)$ is globally passive. In processes satisfying $\Delta\langle A\rangle>0$, the tightest and most informative inequality is found by using $\xi_{-}<0$, which results in the inequality

$$
\Delta\langle A\rangle \leq \frac{1}{\left(-\xi_{-}\right)} \Delta\langle\mathcal{B}\rangle .
$$

Similarly, in processes in which $\Delta\langle A\rangle<0$, one should use $\xi_{+}$, giving

$$
-\Delta\langle A\rangle \leq \frac{1}{\xi_{+}} \Delta\langle\mathcal{B}\rangle
$$


Inequalities (15) and (16) should be viewed as restricting the change of an observable $A$ when compared to the change on another, passive observable. In many of the examples we present, $\mathcal{B}=-\ln \rho_{0}^{\text {tot }}$ will describe a collection of microbaths, so that $\mathcal{B}=\sum_{k} \beta_{k} H_{k}$. Therefore, $\Delta\langle\mathcal{B}\rangle$ contains information about energy changes of subsystems during the process. In contrast, $A$ may describe a fine-grained nonthermal property, for instance, the probability to be in a specific state. Inequalities (15) and (16) then describe how changes in the expectation values of $A$ are restricted by the subsystems energy changes. If $A$ happens to be globally passive then $\Delta\langle A\rangle \geq 0$ and Eq. (15) sets an upper bound on the change in $\langle A\rangle$.

These inequalities include setup-specific information through $\xi_{ \pm}$, which depend on the eigenvalues of $A$ and $\mathcal{B}$. Thus, the method presented here allows us to obtain tighter and more informative inequalities compared to approaches that do not exploit such information, such as Eqs. (10) and (11).

\section{A. Bounds in the presence of constraints and conserved quantities}

Another advantage of the passivity deformation scheme appears in the presence of constrained dynamics and conserved quantities. As explained in the Introduction, the specific form of the interaction may result in disconnected sets of levels that give rise to conserved quantities. As a result, the dynamics is restricted to a subset of allowed unitaries that often does not include the unitary that saturates the bounds that apply to the full set of unitaries. Hence, the bounds are looser than needed, and provide less accurate prediction that may lead, for example, to a degraded heat leak detection capability. In passivity deformation, this can be remedied. Better and tight bounds can be obtained by treating each manifold of states separately. For example, for $\xi_{+}$instead of $\min \left(\xi_{k}>0\right)$, we calculate

$$
\xi_{+}^{\mathrm{int}}=\min \left\{\min \left(\xi_{k \in\left\{l_{1}\right\}}>0\right), \min \left(\xi_{k \in\left\{l_{2}\right\}}>0\right), \ldots\right\}
$$

where, due to the restricted dynamics, the set of states $\left\{l_{i}\right\}$ never interacts with the set $\left\{l_{j \neq i}\right\}$.

As an example, consider two four-level microbaths, initially at inverse temperatures $\beta_{c}=2$ and $\beta_{h}=1$. The energy levels are $E_{c}=\{0,4,8,12\}$ and $E_{h}=\{0,1,2,3\}$. The initial state of the system is therefore described by the density matrix $\rho_{0}^{\text {tot }}=\exp \left(-\beta_{c} H_{c}\right) / Z_{c} \otimes \exp \left(-\beta_{h} H_{h}\right) / Z_{h}$. The unitary evolution is generated by creation-annihilation interactions that couple only the first three levels $H_{\text {int }}=$ $a_{12} b_{12}^{\dagger}+a_{23} b_{23}^{\dagger}+$ H.c., where $a_{i j}\left(b_{i j}\right)$ is the annihilation operator $|i\rangle\langle j|$ in the cold (hot) microbath [see Fig. 4(a)]. As a result, there are several conserved quantities, e.g., the population of the fourth level in each microbath is conserved. Note that the average energy is not conserved since we chose different energy spacings in the two microbaths.

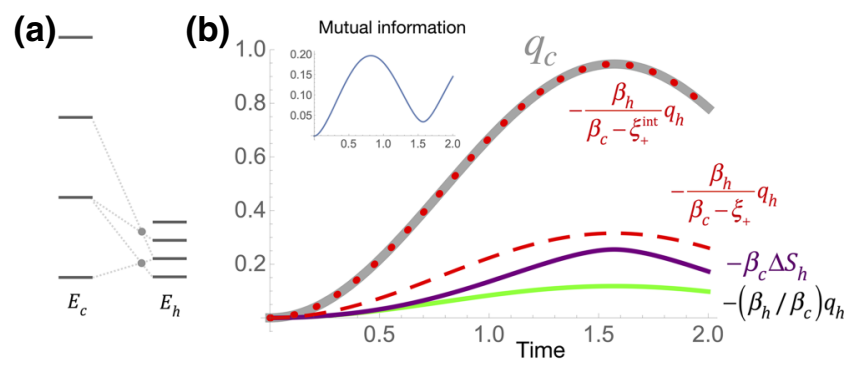

FIG. 4. (a) Two four-level systems (microbaths) with initial inverse temperatures $\beta_{c}, \beta_{h}$ interact via creation-annihilation terms. (b) The gray curve shows the actual change of the average energy of the cold microbath $q_{c}=\Delta\left\langle H_{c}\right\rangle$. The green line depicts bound (10). The bound in Eq. (11) is shown in purple. The unrestricted passivity deformation prediction in terms of heat (dashed red) is tighter than those given in Eqs. (10) and (11), which are based on the increase of entropy. The passivity deformation bound that also uses information about the constraints on the dynamics (dotted red) is tight (analytically). The inset shows that the mutual information (correlation) is not zero in the process. This shows two points of merit for the present framework: (i) it is tight in the presence of correlation and (ii) it can take into account constraints on the dynamics and produce tight bounds despite the constraint.

Thus, the microbaths exchange energy not only with each other but also with the external field that generates $H_{\text {int }}$. At the end of the evolution, the interaction is switched off.

In Fig. 4(b), different bounds on $\Delta\left\langle H_{c}\right\rangle$ are compared to the numerically calculated value of $\Delta\left\langle H_{c}\right\rangle$ (denoted by $q_{c}$ in the figure for brevity). The gray curve represents the exact value of $\Delta\left\langle H_{c}\right\rangle$. The purple line shows the bound imposed by Eq. (11), and the green line shows the bound imposed by Eq. (10). The dashed red line represents the passivity deformation prediction based on Eq. (13), where $\xi_{+}=5 / 8 \beta_{c}$. Exploiting the conserved quantities in this specific interaction we use Eq. (17) and get $\xi_{+}^{\text {int }}=7 / 8 \beta_{c}$. This latter bound $\Delta\left\langle H_{c}\right\rangle \geq\left[\beta_{h} / \beta_{c}(1-7 / 8)\right] \Delta\left\langle H_{h}\right\rangle$ is tight in this example, as can be seen by the red dots.

Interestingly, the bound is tight although there is a nonnegligible correlation between the two objects, as shown in the inset of Fig. 4(b). In Ref. [44] it was indicated that, for large environments, the correlation is not the main mechanism that makes Eq. (11) nontight. It is the deviation from equilibrium in the reservoir that plays a major role. In this example both mechanisms are important. Nevertheless, we see that a bound with $\xi_{+}^{\text {int }}$ is tight despite the deviation from equilibrium in the microbaths. Note that deviation of the bath from equilibrium during the evolution was also treated in Refs. [9,10,37].

To conclude this section, we emphasize that the notion of integrating a constraint on the dynamics in this paper is different from having a second law where some of the quantities satisfy a conservation law. We exploit the constraint (or conservation law) to produce a tighter bound compared to the bound without the 
constraint. The outcome is a bound that has the same observables as the bound without the constraint [e.g., $\Delta\left\langle H_{c}\right\rangle \geq\left\{\beta_{h} / \beta_{c}(1-7 / 8)\right\} \Delta\left\langle H_{h}\right\rangle$ compared to $\Delta\left\langle H_{c}\right\rangle \geq$ $\left(\beta_{h} / \beta_{c}\right) \Delta\left\langle H_{h}\right\rangle$ in the example above], but is tighter and can be saturated despite the restriction on the allowed unitaries. Constraints in the context of the second law, have been addressed in Ref. [45] (quantum systems) and Ref. [46] (classical systems) using information-based quantities rather than observables as in the present paper.

\section{B. Bound-saturating, path-independent operations}

In the previous subsection, we derived a bound of the form $\Delta\langle B(\xi)\rangle \geq 0$, where $B(\xi)=\mathcal{B}+\xi A$ is the sum of a passive and a nonpassive operator, that was valid for a finite range of $\xi$. It is of interest to understand the properties of processes that saturate this bound. This can be further motivated by an analogy with traditional thermodynamics. The Clausius inequality $\Delta S_{\text {sys }}-\int \delta Q / T \geq 0$ is saturated by reversible processes $(Q$ is the heat entering the system while $q$ is the heat entering the reservoir). Reversible processes keep the reservoirs infinitesimally close to equilibrium and do not generate classical correlations and/or entanglement between the system and reservoirs (see Appendix C and Refs. [4,35]). Reversibility constrains the process and enables one to exactly express changes in one element of the setup in terms of changes in the others. For instance, in a reversible process, the change in the entropy of the system (e.g., the engine's working medium) is fully determined by the changes in the energy of the microbaths (heat) $q_{k}: \Delta S_{\text {sys }}^{R}=-\sum_{k} \beta_{k} q_{k}^{R}$ with the $R$ superscript denoting a reversible process. Two very different reversible processes that have the same $\Delta S_{\text {sys }}^{R}$ (they can even involve completely different levels of the system) will have the same "weighted heat" $\sum_{k} \beta_{k} q_{k}^{R}$. In particular, in the special case of a single bath $\Delta S_{\text {sys }}^{R}$ fixes the heat exchanged with the bath and makes it path independent. If the two processes also have the same energy change in the system then the work becomes path independent as well since $W=\Delta F=\Delta\left(S_{\text {sys }}-\beta\left\langle\mathrm{H}_{\text {sys }}\right\rangle\right)$. That is, by specifying the initial density matrix of the system alone, it is possible to uniquely determine how much work and heat were invested to make this change in a reversible manner.

In analogy to the path independence associated with saturating the Clausius inequality, we ask what are the processes that saturate the bound $\Delta\langle B(\xi)\rangle=0$. A trivial way to saturate this bound is to apply processes that leave the initial density matrix unchanged. This does not necessarily imply that $U=I$. If there are degeneracies in $\rho_{0}^{\text {tot }}$ then there is a family of unitaries that only mix states within each degenerate subspace, and for any mixture of such unitaries, $\rho_{f}^{\text {tot }}=\sum_{k} p_{k} U_{k} \rho_{0}^{\text {tot }} U_{k}^{\dagger}=\rho_{0}^{\text {tot }}$. Such operations trivially lead to $\Delta\langle B(\xi)\rangle=0$ (as for any other expectation value). Thus, these trivial degeneracies are of no interest to us.
A nontrivial way of saturating the bound can be found when the operator $B(\xi)=\mathcal{B}+\xi A$ has degeneracies that are different from the trivial degeneracies of $\mathcal{B}$. Interestingly, these degeneracies are guaranteed to appear at $\xi=$ $\xi_{ \pm}$. These degeneracies facilitate nontrivial processes that redistribute population between these degenerate states of $B(\xi)$, while keeping the expectation value $\langle B(\xi)\rangle$ fixed (assuming that no other operation takes place). For a general mixture of unitaries, we have inequalities (15) and (16). However, if we restrict the dynamics to be a mixture of unitaries that only couple the states that become degenerate at $\xi_{ \pm}$, the inequality can be replaced by the equality

$$
\sum_{k} \beta_{k} q_{k}^{\mathrm{BSP}}=-\xi_{ \pm} \Delta\langle A\rangle_{\mathrm{BSP}}
$$

where the index BSP (bound-saturating process) indicates that only unitaries limited to this $B\left(\xi_{ \pm}\right)$degenerate subspace are included. Crucially, an interaction between nontrivially degenerate states leads to $\rho_{f}^{\text {tot }} \neq \rho_{0}^{\text {tot }}$ since these states are associated with different initial probabilities.

The implications of Eq. (18) are similar to the familiar reversible path independence mentioned earlier: knowing that change in $\langle A\rangle$ was created by a BSP, fixes the value of $\sum_{k} \beta_{k} q_{k}$ regardless which BSP was actually used. Note that the BSPs for $\xi_{-}$and $\xi_{+}$are necessarily different from each other since otherwise there will be two conflicting predictions on $\sum_{k} \beta_{k} q_{k}$.

Interestingly, since $\mathcal{B}(\xi)$ and $A$ in Eq. (18) have different eigenvalue ordering when written in the same basis, they cannot be minimized at the same time (although they commute). Thus, there is a generic trade-off between saturating the $B_{\xi}$ bound and performance (minimizing or maximizing $\langle A\rangle)$. In contrast to the familiar power-efficiency trade-off in heat machines, the present trade-off has nothing to do with time and adiabaticity. It refers to the total accumulated effect and time plays no role here.

It is instructive to highlight a key difference between reversible operations and BSP. While BSPs are guaranteed to exist whenever $\xi_{-}$or $\xi_{+}$are different from zero, reversible operations such as isotherms cannot be implemented with small environments. There are two reasons for this: (1) the microbaths develop non-negligible correlation; (2) due to their small heat capacity, the microbaths do not remain locally in equilibrium with their original temperature. In contrast, the BSPs are standard unitary operations that can be implemented with suitable control fields. That is, in microscopic setups the dynamics is thermodynamically irreversible [Eq. (11) is not saturated]. Nevertheless, the BSP saturates the passivity deformation bounds despite the thermodynamic irreversibility.

In summary, by examining the structure of the passive operators of the form $B\left(\xi_{ \pm}\right)$and restricting the allowed unitaries to those associated with the emerging $\xi_{ \pm}$ degeneracies we identify nontrivial processes that saturate 
the passivity-based bounds (15) and (16). The path independence associated with the equality shows some resemblance to reversible processes. However, the reasons for the saturation of the bound are quite different in both instances. Proximity to equilibrium during the dynamics in one case, and a restriction to evolution in a degenerate subspace in the other. Finally, we wish to emphasize that passivity deformation inequalities (with the extremal values $\xi_{ \pm}$) can always be saturated by some unitary evolution, even if the environments are very small. This addresses the unattainability issue of Eqs. (10) and (11), and global passivity.

\section{Illustrative examples}

In this section, we present several examples that demonstrate how to obtain inequalities with interesting physical interpretation using the passivity deformation approach. These examples are intentionally chosen to be elementary and involve just a few particles. We aim to show how this method works in the simplest setups and what kind of results it can provide. Some of the spin-based examples shown here can be extended to any number of spins in the environment. An additional set of examples will be presented later, after introducing a graphical approach to passivity deformation.

\section{Performance of an exotic heat machine}

Consider the machine described in Fig. 1(c), namely a two-spin system that can be manipulated by interacting with a microbath. To keep the plot simple and tractable, we first use a two-spin environment (microbath). The goal of the setup is to implement a protocol of interaction with the environment that will make the two spins of the system as correlated as possible. Specifically, we wish the system spins to be in the $|00\rangle_{\text {sys }}$ state or in the $|11\rangle_{\text {sys }}$ state. The Hamiltonian of the setup is $H=H_{\text {sys }}+$ $H_{\text {env }}+H_{\text {int }}(t)$, where $H_{\text {sys }}=\omega \sigma_{1}^{z}+\omega \sigma_{2}^{z}, H_{\text {env }}=\omega \sigma_{3}^{z}+$ $\omega \sigma_{4}^{z}$. Here $H_{\text {int }}(t)$ depends on the protocol used for the correlation enhancement. Initially, $H_{\text {int }}(0)=0$, and the initial inverse temperature of the whole setup is $\beta=1 / 2$. Hence, the initial state is $\rho_{0}^{\text {tot }}=e^{-\beta H} / \operatorname{tr}\left[e^{-\beta H}\right]$.

The probabilities to be in a certain set of states is given by expectation values of the projection operator to this set of states. In the present case the goal is to increase the expectation value of the projector $A=|00\rangle\left\langle\left. 00\right|_{\text {sys }}+\right.$ $|11\rangle\left\langle\left. 11\right|_{\text {sys }}\right.$, since $P_{\text {same }}=P(11)+P(00)=\operatorname{tr}(\rho A)=\langle A\rangle$. What are the limitations on this class of processes? Which resources must be invested to generate the desired output? Using the form $B_{\xi}=\mathcal{B}+\xi \omega A$, and employing Eq. (16), we find that $\xi_{-}=-\beta$. As a result, we obtain the bound

$$
\Delta P_{\text {same }} \leq W / \omega,
$$

where $W=\Delta\left\langle H_{\text {sys }}+H_{\text {env }}\right\rangle$ is the work done on the setup during the process. We conclude that our ability to realign
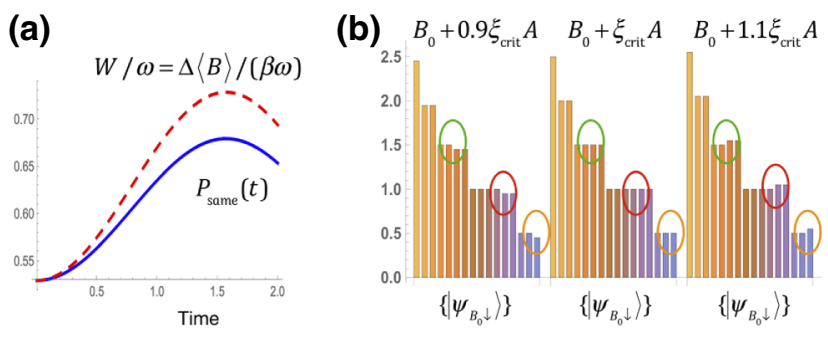

FIG. 5. (a) The blue curve shows the change in the correlation of the two spins in the machine illustrated in Fig. 1(c). Passivity deformation predicts that the (scaled) work (dashed red) is always larger than the correlation creation (blue). (b) The bar plots show the emergence of degeneracies before, at, and after the critical point $\xi_{-}$. Unitary operation between states in the same ellipse exhibit path-independent behavior.

the system's spins is directly bounded by how much work we invest. Note that this result holds for any $\beta$ and any $\omega$. Using the method described in Appendix B, we find the unitary that maximizes $\langle A\rangle$. In Fig. 5(a) we show that in accordance to the passivity construction prediction, $\Delta P_{\text {same }} \leq W / \omega$, the work (normalized by $\omega$ ) that has to be invested (red line) is larger than the increase in the probability of the spins to be in the same orientation. As explained at the end of Sec. III B, the saturation of the passivity deformation bounds typically conflicts with achieving the maximal performance. The unitary that maximizes $\Delta P_{\text {same, }}$, which was used in this example, is different from the BSP unitary that saturates the bounds. Hence, the bound cannot be tight in this example.

In Fig. 5(b) we show the emergence of new degeneracies at $\xi=\beta$. If the dynamics is restricted to unitaries that mix only states inside each ellipse, then it is guaranteed that Eq. (19) will reach equality. That is, the amount of work will not depend on which BSP transformation we applied, only on the change in the correlation observable $\Delta P_{\text {same}}$, i.e., there is path independence for BSP operations.

We point out that inequalities such as those in Eq. (10) or (11) are not suitable for setting performance bounds for such machines as they do not contain the changes in the fine-grained observable $A$. This example illustrates the utility of our approach to quantifying the performance limits of such exotic heat machines.

Using the graphical tool presented in Sec. IV, it is possible to show that Eq. (19) holds for any number of environment qubits (provided that the product $\beta \omega$ is the same for all qubits). Furthermore, in systems with more than two qubits, the creation of correlation between other sets of states can be studied in the same way.

\section{Bounds on the system-environment covariance in dephasing dynamics}

To show a more quantum aspect of our approach, we consider dephasing dynamics. In our previous work [2] we 


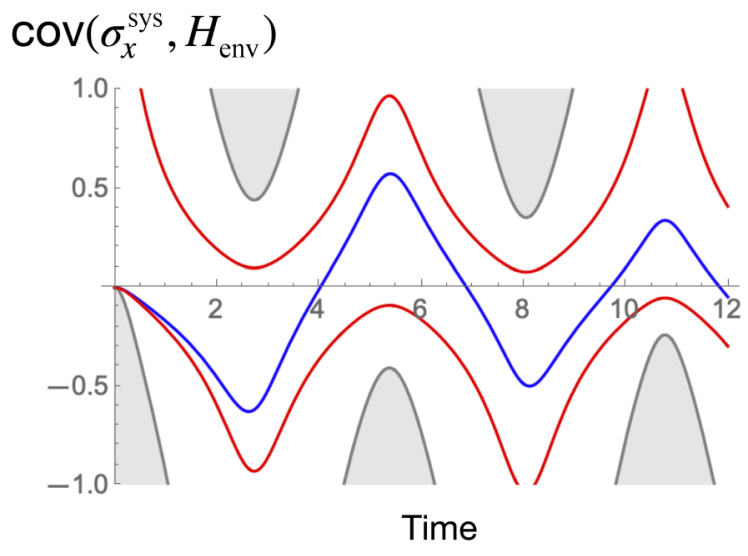

FIG. 6. The dynamics of the normalized covariance between the polarization of an initially coherent spin and the energy of a dephasing environment composed of three thermal spins. The exact dynamics is shown in blue and the gray areas are the forbidden zones according to the global passivity bound $\Delta\left\langle B^{2}\right\rangle \geq 0$. Using the passivity deformation framework, we find significantly tighter bounds (red).

obtained a bound on the covariance between the coherence of a system and the energy of its dephasing environment, i.e., $\left\langle\sigma_{x}^{\text {sys }} H_{\text {env }}\right\rangle-\left\langle\sigma_{x}^{\text {sys }}\right\rangle\left\langle H_{\text {env }}\right\rangle$, where $H_{\text {env }}=\sum_{j=1}^{3} \sigma_{z}^{(j)}$ is the Hamiltonian of the environment. The Hamiltonian of the system is $H_{\text {sys }}=\sigma_{z}^{\text {sys }}$. The system was a spin with some initial coherence in the energy basis, and it interacted with a three-spin microbath (environment) via an interaction of the form $H_{\text {int }}=\sum_{j=1}^{3} \gamma_{j} \sigma_{z}^{\text {sys }} \otimes \sigma_{z}^{(i)}$. Here $\gamma_{i}=\{0.7,0.5,0.3\}$ is a set of couplings that represents the case where some environment spins are further away from the system. The system Hamiltonian is $H_{\text {sys }}=\sigma_{z}^{\text {sys }}$, and the initial state of the setup is $\rho_{0}^{\text {tot }}=\exp \left(-\beta_{x} \sigma_{x}^{\text {sys }}-\beta H_{\text {env }}\right) / Z$ with $\beta_{x}=\beta=3$. Note that the state of the system is not thermal since the system Hamiltonian is $\sigma_{z}^{\text {sys }}$ and not $\sigma_{x}^{\text {sys }}$. From the initial density matrix we obtain $\mathcal{B}=\beta_{x} \sigma_{x}^{\text {sys }}+$ $\beta H_{\text {env }}+\ln Z$.

In Ref. [2] we used the global passivity of $\mathcal{B}^{2}$ to set a bound on $\left\langle\sigma_{x}^{\text {sys }} H_{\text {env }}\right\rangle$. Here we look for a tighter inequality by constructing $B(\xi)=\mathcal{B}^{2}+\xi \sigma_{x}^{\text {sys }}$ and studying for which $\xi$ values $B(\xi)$ is globally passive. The gray regions in Fig. 6 show the bounds obtained in Ref. [2] based on $\Delta\left\langle\mathcal{B}^{2}\right\rangle \geq 0$, and the red curves show the passivity deformation bounds $\Delta\left\langle B\left(\xi_{-}\right)\right\rangle \geq 0$ with $\xi_{-}=-9$. See Ref. [2] for the technique used to derive the upper bounds. Clearly, the passivity deformation bound is closer to the actual dynamics of the covariance (blue) compared to the global passivity bound.

\section{Demon detection through violation of passivity deformation bounds}

One of the ways in which inequalities of the type derived here can be useful is through their violation, which indicates that some of the assumptions made on the dynamics of the setup are not valid. In Ref. [2] we used this idea for detecting the presence of Maxwell-like demons that may tamper with the dynamics. The demons that were considered were too subtle to be detected by the second law. Nevertheless, they were detectable by the violation of some of the global passivity inequalities $\Delta\left\langle\mathcal{B}^{\alpha}\right\rangle \geq 0$. As it turns out, although the inequalities $\Delta\left\langle\mathcal{B}^{\alpha}\right\rangle \geq 0$ are more sensitive to the presence of demons compared to Eq. (10), they may still fail to detect a demon whose operation is sufficiently weak. This raises the key question regarding the existence of more sensitive thermodynamiclike tests (inequalities) that can detect the tampering of these lazier demons.

Consider two initially uncoupled microbaths at different temperatures, i.e., $\rho_{0}^{\text {tot }}=\exp \left(-\beta_{c} H_{c}\right) / Z_{c} \otimes \exp \left(-\beta_{h} H_{h}\right) /$ $Z_{h}$. In the absence of external work, the second law assures us that subsequent evolution will result in energy transfer from the hot to the cold microbath. If these two microbaths are well isolated from the rest of the world, and there is no Maxwell's demon, the evolution is unitary, $\rho_{f}^{\text {tot }}=U \rho_{0}^{\text {tot }} U^{\dagger}$. For simplicity, we consider a demon that is operating on the setup at the end of the unitary evolution. The demon applies feedback that depends on the state of the setup, resulting in dynamics described by a Kraus map $\tilde{\rho}_{f}^{\text {tot }}=\sum_{k} U_{k} \Pi_{k} \rho_{f}^{\text {tot }} \Pi_{k} U_{k}^{\dagger}$, where $\Pi_{k}$ denotes a projection into the subspace that matches the measurement outcome $k$. Passivity-based inequalities are not guaranteed to hold under such evolution.

There are two ways to make demon detection more challenging. The first is to apply mild operations. That is, to use demon (feedback) operations $U_{k}$ that are very close to the identity. The second option is to apply the feedback with some probability $p$, and with probability $1-p$, do nothing so that $\tilde{\rho}_{f}^{\text {tot }}=p \sum_{k} U_{k} \Pi_{k} \rho_{f}^{\text {tot }} \Pi_{k} U_{k}^{\dagger}+(1-p) \rho_{f}^{\text {tot }}$. Consequently, for the same feedback operations $U_{k}, p=0$ corresponds to demon-free evolution while $p=1$ is the standard Maxwell demon (that violates the second law). A demon with a low enough value of $p$, so that $\beta_{c} \Delta\left\langle H_{c}\right\rangle+$ $\beta_{h} \Delta\left\langle H_{h}\right\rangle \geq 0$ and Eq. (10) cannot be used to detect it, is called a lazy demon.

In the lazy demon scenario considered in Ref. [2] it was found that the optimal detection using the $\Delta\left\langle\mathcal{B}^{\alpha}\right\rangle$ family of inequalities takes place at $\alpha \simeq 2.56$ (this value is not universal). This suggests that higher $\alpha$ values are not necessarily better, and that noninteger values of $\alpha$ can have a practical advantage. Most importantly, inequality (10) $(\alpha=1)$ fails to detect this demon.

We return to this example and test whether one can find even more sensitive inequalities using the approach developed here. In the example used in Ref. [2] both microbaths were two-spin systems, with $H_{c}=\sigma_{z}^{(1)}+\sigma_{z}^{(2)}$ and $H_{h}=\sigma_{z}^{(3)}+\sigma_{z}^{(4)}$. The initial inverse temperatures were chosen to be $\beta_{c}=2 / 3$ and $\beta_{h}=0.4$. To derive passivity-deformation-based inequalities, we assume that 
the dynamics in the absence of a demon is unitary and construct the operator $B_{\sigma_{z}^{(3)}}(\xi)=\beta_{c} H_{c}+\beta_{h} H_{h}+\xi \sigma_{z}^{(3)}$. Using Eqs. (15) and (16), we find that the range of $\xi$ values for which this operator is passive is between $\xi_{-} \approx-0.266$ and $\xi_{+} \approx 0.133$.

We now consider a process that involves evolution with an "all-to-all" interaction between the spins $H_{I}=$ $\sum_{i>j} \sigma_{+}^{(i)} \sigma_{-}^{(j)}+\sigma_{-}^{(i)} \sigma_{+}^{(j)}$. After the evolution, the demon is awake with probability $p$. If it is awake and the system is in the state $|1100\rangle$, it replaces it with the state $|0011\rangle$. In all other cases, the demon does nothing. In this example, a demon that operates 0.56 of the time or more will violate Eq. (10). In Ref. [2] it was shown that a demon that operates only 0.48 of the time (or more) will violate the inequality $\Delta\left\langle\mathcal{B}^{\alpha}\right\rangle \geq 0$ (with $\alpha=2.56$ ). Crucially, using passivity deformation, we find that a demon that operates more than 0.289 of the time will violate the inequality $\Delta\left\langle B_{\sigma_{z}(3)}\left(\xi_{-}\right)\right\rangle \geq 0$ derived here. Note that the inequality $\Delta\left\langle\beta_{c} H_{c}+\beta_{h} H_{h}\right\rangle \geq 0$ cannot detect the demon while the passivity deformation inequality $\Delta\left\langle\beta_{c} H_{c}+\beta_{h} H_{h}+\right.$ $\left.\xi_{-} \sigma_{z}^{(3)}\right\rangle \geq 0$ can detect the demon without resorting to higher-order moments. Nevertheless, by using the passive operator $\left[B_{\sigma_{z}^{(3)}}\left(\xi_{-}\right)\right]^{\alpha}$, the detection threshold further improves to $p>0.25$ at $\alpha=2.64$. Hence, the passivity deformation approach leads to more sensitive detection compared to global passivity.

This example, as well as the previous examples studied in this section, demonstrate that inequalities (15) and (16) are tighter, and therefore more informative than inequality (10). Moreover, they are tight even in the presence of small environments and correlation buildup. This comes at a certain cost. To derive these new bounds, one has to exploit system-specific information about the eigenvalues of relevant operators, and on the initial state of the setup. This setup dependence of $\xi$ is also the reason for the tightness of the bounds with respect to Eqs. (10) and (11) - passivity deformation bounds are constructed to handle only the scenarios that can actually take place in the setup of interest (e.g., see the discussion on constraints and conserved quantities). In many modern experimental setups such as superconducting qubits, trapped ions, or optical lattices, the initial state and the Hamiltonian are known. Hence, the inequalities studied here are well suited for the description of quantum processes in such setups.

\section{ADDITIONAL INSIGHTS FROM A GRAPHICAL REPRESENTATION OF PASSIVITY DEFORMATION}

In this section, we explore a more visual and intuitive method for obtaining passivity-deformation-based inequalities. The basic idea is to start with the globally passive operator $\mathcal{B}=-\ln \rho_{0}^{\text {tot }}$, and deform it to a new operator $\widetilde{\mathcal{B}}$, by shifting some of its levels (eigenvalues) using a set of rules that ensure that the resulting operator is globally passive. As a result, the subsequent evolution will satisfy

$$
\Delta\langle\widetilde{\mathcal{B}}\rangle \geq 0
$$

Although we later consider more complicated scenarios, let us start with the basic scenario of two uncorrelated microbaths for which

$$
\mathcal{B}=\beta_{h} H_{h} \otimes I_{c}+I_{h} \otimes \beta_{c} H_{c},
$$

where we have explicitly denoted the identity operators in each subspace. We also dropped an additive constant, arising from the normalization of $\rho_{0}^{\text {tot }}$, that would not affect the resulting inequality. Since the two terms commute, the eigenvalues of $\mathcal{B}$ have the form $\beta_{c} E_{\lambda}^{(c)}+\beta_{h} E_{\nu}^{(h)}$ with $E_{\lambda}^{(c)}\left(E_{v}^{(h)}\right)$ denoting the eigenvalues of $H_{c}\left(H_{h}\right)$. It is most useful to plot this spectrum using "floors" and "ladders," as shown in Fig. 7. First, we select one of the microbaths, e.g., the cold one, to set the floors and plot the level $\beta_{c} E_{\lambda}^{(c)}$ with an increasing sideways shift for each level so that a staircase shape is obtained (the stairs may be uneven). Next, on each floor, we set a ladder of the hot levels $\beta_{h} E_{v}^{(h)}$. The floors are not actual levels of $\mathcal{B}$, but merely a reference for the ladders. The positioned ladders constitute the actual levels of $\mathcal{B}$.

With the sideways shift, it is easy to read off $\mathcal{B}$ from the plot. Without the shift, only the spectrum values are accessible and it is difficult to note that it has the form $\beta_{h} H_{h} \otimes I_{c}+I_{h} \otimes \beta_{c} H_{c}$ if $\beta_{c} H_{c}$ and $\beta_{h} H_{h}$ are not known a priori. Furthermore, this representation is very useful for understanding how the spectrum changes if we continuously change some of the parameters, for example, the temperature of one of the elements. Note that the ladders may overlap in height [Fig. 7(a)] or be separated from each other [Fig. 7(b)]. As explained later, this separation has physical implications. It is straightforward to extend the plot to multiple microbaths in an iterative way. For example, for three microbaths, start by plotting two as before,

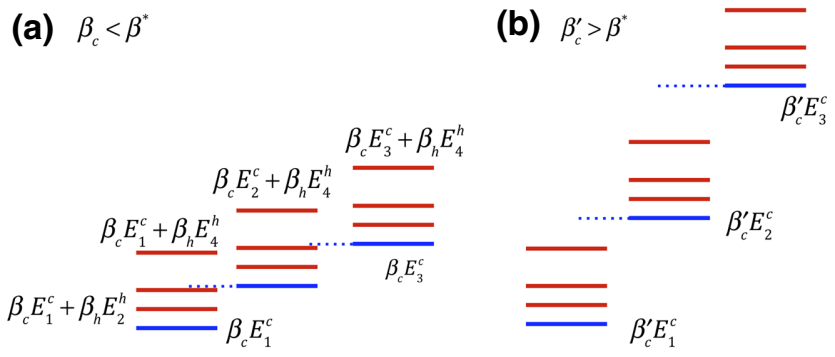

FIG. 7. Plotting the spectrum of a globally passive operator of the form $\beta_{c} H_{c}+\beta_{h} H_{h}$. For high enough temperatures $T_{c}$ (low $\beta_{c}$ ), the hot manifolds overlap (a), while for cold enough temperatures (high $\beta_{c}$ ), they do not (b). 
then consider the resulting plot as "floors" and use the third microbath as a ladder.

Let us define a new operator $\widetilde{\mathcal{B}}$ that has the same eigenstates as $\mathcal{B}$, i.e., $[\widetilde{\mathcal{B}}, \mathcal{B}]=0$, but can have different eigenvalues. To ensure that the new operator $\widetilde{\mathcal{B}}$ stays globally passive, we want it to have the same order of eigenvalues as $\mathcal{B}$. Individual levels can be shifted up or down and, as long as they do not cross any other levels, the order of the new eigenvalues of $\tilde{\mathcal{B}}$ will still be the same as that of $\mathcal{B}$. Crossing levels is equivalent to level permutation that changes the order and breaks global passivity. Since degenerate levels of $\mathcal{B}$ have no order between themselves, we can split and move them until they touch another level they were not originally degenerate with. Thus, the idea of passivity deformation can be stated as follows.

\section{Passivity deformation.}

An operator $\tilde{\mathcal{B}}$ created from $\mathcal{B}=-\ln \rho_{0}^{\text {tot }}$ by applying the following operations

(1) moving up and down levels without crossing other levels,

(2) splitting levels that were degenerate in $\mathcal{B}$, is globally passive, i.e., it satisfies $\Delta\langle\tilde{\mathcal{B}}\rangle \geq 0$ for any proto$\operatorname{col} \rho_{f}^{\text {tot }}=\sum_{k} p_{k} U_{k} \rho_{0}^{\text {tot }} U_{k}^{\dagger}$.

If the dynamics does not mix all levels (i.e., there are dynamical constraints and conserved quantities) then we have the following more flexible set of rules.

Passivity deformation under restricted dynamics.

Let the dynamics be composed of a mixture of unitaries $U_{k}^{\prime}$ that do not mix two specific levels $m$ and $l$ (levels of the whole setup), i.e., $\left\langle m\left|U_{k}\right| l\right\rangle=\left\langle l\left|U_{k}\right| m\right\rangle=0$. An operator $\tilde{\mathcal{B}}$ created from $\mathcal{B}$ using rules (1) and (2) above, as well as the rule that

(3) crossing levels $m$ and $l$ is allowed (provided that no other levels are crossed in the process),

is globally passive, i.e., it satisfies $\Delta\langle\tilde{\mathcal{B}}\rangle \geq 0$ for any protocol generated by the restricted dynamics $\rho_{f}^{\text {tot }}=\sum_{k} p_{k} U_{k}^{\prime} \rho_{0}^{\text {tot }} U_{k}^{\prime \dagger}$.

The additional third rule can be stated using a conservation law. In the case of a conserved quantity $Q$, crossing should be avoided only between levels that have the same value of $Q$.

We emphasize that these deformations are by no means physical operations we execute on the setup. They are just a mathematical technique for finding new globally passive operators and new inequalities. The physical interpretation of the resulting inequalities depends on the passive operators $\tilde{\mathcal{B}}$ that can be identified. The interpretation does not have to be thermodynamic in nature (e.g., involving energies of subsystems).
As shown later in Sec. IVC, this deformation recipe [rules (1)-(3)] is not limited to objects that are initially in a Gibbs state or to uncorrelated objects. Next, we study several key examples that illustrate the value of the passivity deformation approach and the graphical representation in particular.

\section{A. Addressing the ultracold catastrophe}

\section{Ultracold environments and nonoverlapping ladders}

As an example for a process involving a very cold environment, let us consider once again a setup composed of two microbaths that are initially thermal with inverse temperatures $\beta_{c}$ and $\beta_{h}$. Then, the setup is driven by a process described by a mixture of unitaries. If the initial temperature $1 / \beta_{c}$ is too low, no unitary process (or a mixture of unitaries) can cool the cold microbath, irrespective of the amount of invested work. That is, a refrigerator cannot be implemented in the given setup. As shown below, this is a rather general result. We term such environments "ultracold environments."

By construction, the spacings between the floors [blue lines in Fig. 7(a)] are proportional to $\beta_{c}$. Hence, when decreasing the initial temperature, the spacings between the blue levels expand. Yet, the spacings in the red ladder are not affected by the change in $\beta_{c}$. Next, we assume that the spectral range of the hot microbath $\omega_{h}^{\max }=\max \left(E^{h}\right)-$ $\min \left(E^{h}\right)$ is finite. Under this assumption, it follows that, for large enough $\beta_{c}$, the ladders will not overlap with each other, as shown in Fig. 7(b). The condition for nonoverlapping ladders is

$$
\begin{gathered}
\beta_{c} \geq \beta_{c}^{\star}, \\
\beta_{c}^{\star} \doteq \beta_{h} \omega_{h}^{\max } / \omega_{c}^{\min },
\end{gathered}
$$

where $\omega_{c}^{\min }=\min \left(E_{n+1}^{c}-E_{n}^{c}\right)$ is the minimal, nonzero energy gap in the cold bath Hamiltonian. Physically, this condition is met when the initial temperature of the cold microbath is "sufficiently cold." Equation (22) implies that this scale of coldness is not an intrinsic and objective property of the cold environment, but a property with respect to the other systems it potentially interacts with (the hot microbath). Note that in this regime $\beta_{h}$ can be very large as well, as long as Eq. (22) is satisfied. Next, the relation between no cooling and nonoverlapping ladders is outlined.

\section{No cooling in the ultracold regime}

Considering the two-microbath scenario above, inequality (10) constrains the possible changes in the energies of the hot and cold subsystems. Yet, it does not, a priori, determine the sign of $\Delta\left\langle H_{c}\right\rangle$ that may, in principle, depend on the selected interactions. 
Next, we assume that the cold environment is ultracold and that the no-overlap condition (22) holds. Since the ladders do not overlap, according to the passivity deformation rules presented earlier, one can freely expand the distances between them (no levels will be crossed). We uniformly increase the distance between them by replacing $\beta_{c}$ by some fictitious $\beta_{c}^{\prime} \geq \beta_{c}$. Since this a legitimate deformation, it holds that

$$
\beta_{c}^{\prime} \Delta\left\langle H_{c}\right\rangle+\beta_{h} \Delta\left\langle H_{h}\right\rangle \geq 0 .
$$

We remind the reader that this is not a physical change in the system and that the initial temperature is still $\beta_{c}$ and not $\beta_{c}^{\prime}$. Yet, we obtained a new inequality (24) by doing this deformation. Taking the limit $\beta_{c}^{\prime} \rightarrow \infty$ we conclude that

$$
\Delta\left\langle H_{c}\right\rangle \geq 0 \quad \text { for } \beta_{c} \geq \beta_{c}^{\star},
$$

i.e., there is no refrigerator that can exploit the given hot environment, to cool the given ultracold environment. Such behavior is known for specific Otto engines coupled to Markovian environments [47-49]. Here we have used passivity deformation to show that this is a generic property of microbaths and not of a specific machine. Even in complicated machines that involve quantum nonadiabatic couplings that are too complicated to be solved analytically, the conclusion on the lack of cooling window for $\beta_{c} \geq \beta_{c}^{\star}$ still holds. Note that there was no restriction on the applied unitary, so it is possible to add a local unitary on the cold microbath at the end of the evolution that brings the cold microbath to its passive state. Thus, even residual coherence cannot be utilized to overcome the $\Delta\left\langle H_{c}\right\rangle \geq 0$ result for $\beta_{c} \geq \beta_{c}^{\star}$.

\section{Handling the zero-temperature limit}

As explained above, when $\beta_{c} \rightarrow \infty$, the hot (finite) ladders are infinitely separated from each other [see Fig. 7(b)]. According to the rules of passivity deformation described above, we now deform the operator $\mathcal{B}$ into a new operator $\widetilde{\mathcal{B}}$ by shrinking the distance between the ladders (but not shrinking the ladders themselves). As a result, the new operator now has a fictitious $\beta_{c}^{\prime}<\infty$. According to the rules of passivity deformation, $\beta_{c}^{\prime}$ cannot be arbitrarily small. We must stop at the first time the ladders cross. This happens at

$$
\beta_{c}^{\prime}=\beta_{c}^{\star}=\beta_{h} \omega_{h}^{\max } / \omega_{c}^{\min } .
$$

Thus, it holds that in this setup

$$
\beta_{c}^{\star} \Delta\left\langle H_{c}\right\rangle+\beta_{h} \Delta\left\langle H_{h}\right\rangle \geq 0
$$

or, alternatively,

$$
\frac{1}{\omega_{c}^{\min }} \Delta\left\langle H_{c}\right\rangle+\frac{1}{\omega_{h}^{\max }} \Delta\left\langle H_{h}\right\rangle \geq 0 .
$$

This form appears to be temperature independent; however, it is valid only if the real initial cold temperature of the bath $1 / \beta_{c}$ is smaller than $\omega_{c}^{\min } /\left(\beta_{h} \omega_{h}^{\max }\right)$. Equations (27) and (28) provide a more refined prediction compared to Eq. (10) or (11), which only determines the sign of $\Delta\left\langle H_{c}\right\rangle$ via $\Delta\left\langle H_{c}\right\rangle \geq 0$. We now have a meaningful relation between the energy changes in the cold microbath and the hot microbath. In particular, according to Eq. (28), the efficiency of an engine exploiting these two environments is limited by $\eta \leq 1-\beta_{h} / \beta_{c}^{\star}=1-\omega_{c}^{\min } / \omega_{h}^{\max }$, while the prediction of Eq. (10) for $\beta_{c} \rightarrow \infty$ is $\eta \leq 1-$ $\beta_{h} / \beta_{c}=1$, which provides no useful information. The efficiency $1-\beta_{h} / \beta_{c}^{\star}=1-\omega_{c}^{\min } / \omega_{h}^{\max }$ corresponds to an Otto engine operating between the two levels with the smallest nonzero energy gap in the cold bath and the two most separated levels in the hot bath. This process will saturate the revised bound (27). Yet, we emphasize that in the ultracold limit the Otto efficiency $1-\omega_{c}^{\min } / \omega_{h}^{\max }$ limits all possible protocols for work extraction from these two baths.

We should clarify that in this scenario there is no machine that runs a periodic protocol and achieves some steady-state operation. Instead, there is a direct nonenergy-conserving interaction between the two microbaths. Yet this interaction may cool (and consume work) or harvest some work (an engine). In the current setup, there is no subsystem that acts as a working medium that can store part of the energy. As a result, the efficiency remains $W / Q_{h}$ even if a single-shot nonperiodic drive is applied. Finally, for readers who are familiar with microscopic engines, we point out that small environments cannot support the isotherms needed for the Carnot machines (see the discussion about reversible processes in Sec. III B). Thus, for small environments, Carnot machines cannot be implemented regardless of how slow they operate.

To numerically illustrate our findings, we apply the passivity deformation approach to the spin-oscillator setup studied in Ref. [50]. The Hamiltonian is

$$
\begin{aligned}
H & =H_{\mathrm{osc}}+H_{\mathrm{spin}}+H_{\mathrm{int}} \\
& =\omega a a^{\dagger}+\Omega \sigma \sigma^{\dagger}+g\left(a^{\dagger}+a\right)\left(\sigma^{\dagger}+\sigma\right),
\end{aligned}
$$

where initially the oscillator is in some cold temperature $T_{\text {osc }}$, and the excited state population of the spin is 0.1 . The two are initially uncorrelated. The goal is to get a bound on the heat flows that enters the oscillator $q_{\mathrm{osc}}=\Delta\left\langle H_{\mathrm{osc}}\right\rangle$ using quantities that involve only the system. Employing Eq. (28) to the present scenario yields the following lower bound on $\Delta\left\langle H_{\text {osc }}\right\rangle$ :

$$
\Delta\left\langle H_{\mathrm{osc}}\right\rangle \geq \frac{\omega}{\Omega} \Delta\left\langle H_{\mathrm{spin}}\right\rangle .
$$

In Fig. 8 the numerically calculated value of $\Delta\left\langle H_{\text {osc }}\right\rangle$ is depicted in black, and the prediction $T_{\text {osc }} \Delta S_{\text {sys }}$ obtained from Eq. (11) is shown in red. The bound derived in 


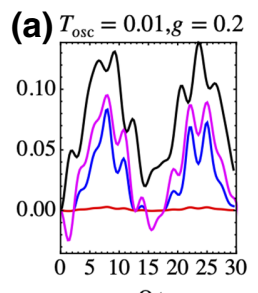

$\Omega t$

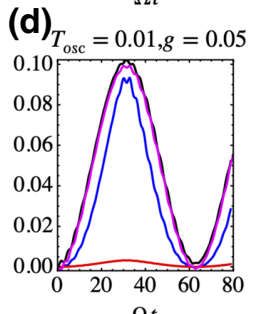

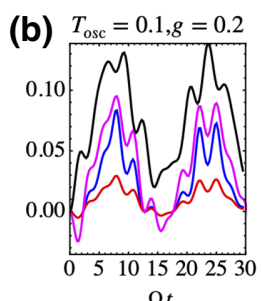

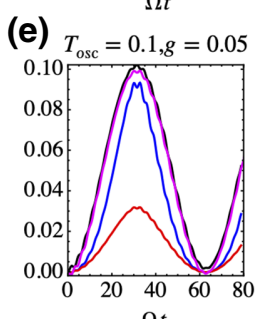

$\Omega t$

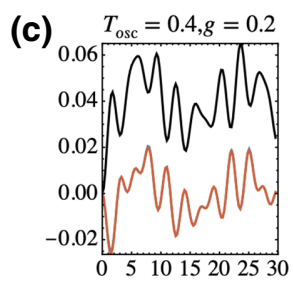

$\Omega t$

(f)

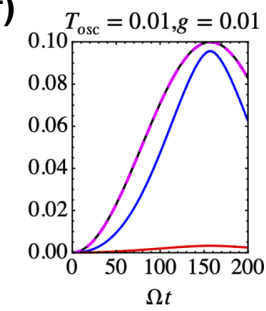

FIG. 8. Various bounds on the change in energy of a cold oscillator that is coupled to a spin. In black is the numerically calculated energy change of the oscillator. The red line represents bound (11). The blue line shows the bound obtained in Ref. [50] and the magenta line shows our passivity deformation bound (30). In all plots the passivity deformation bound is the tightest. The values of the coupling constant and the initial temperature of the oscillator are given in each plot. In Fig. 8(f) the coupling strength is very weak, $g=0.01$. As appears in this figure, in the limit $g \rightarrow 0$ the passivity deformation bound becomes tight while the other bounds do not.

Ref. [50] is shown in Blue, and the magenta line corresponds to the passivity deformation bound.

In the top row of Fig. $8, g=0.2$; in Figs. $8(\mathrm{~d})$ and $8(\mathrm{e})$, $g=0.05$; and in Fig. 8(f), $g=0.01$. In addition, $\omega=\Omega=$ 1 , as taken in Ref. [50]. The initial temperatures of the oscillators from left to right in Fig. 8 are $T=0.01, T=0.1$, and $T=0.4$. Interestingly, as shown in Fig. $8 \mathrm{c}$, for hot temperatures all bounds become equal (the blue and magenta lines are behind the red). The passivity deformation bound is the tightest at all times in all plots. In Fig. 8(f) we show that, for an even weaker coupling than that tested in Ref. [50], the passivity deformation bounds approach the actual values. We observe that, as the coupling gets weaker (and the evolution time is larger to compensate for that), the passivity deformation becomes tighter and tighter. On the other hand, the bound presented in Ref. [50] does not become tighter and can be seen by comparing the blue line in Figs. 8(d) and 8(f).

Finally, we compare our approach to a result from another paper [51], where an environment with several spins $(1,3$, or 5$)$ interacts with a single spin. The bounds studied in Ref. [51] show an improvement over Eq. (11) and a bound studied in Ref. [51]. Yet none of the presented bounds are tight. We find that, for the case in which the initial state of the system is hot (Fig. 3 of Ref. [51]), our bound is tight. When the initial state is pure (excited state) (see Fig. 4 of Ref. [51]), our bound is tight provided that the restricted dynamics passivity deformation scheme described in Sec. III A is employed.

\section{B. Information erasure and the energetic cost of polarization creation}

One of the merits of the passivity deformation approach is that it can be used to obtain bounds on various finegrained observables and not only on the average energy and the entropy. As an example, consider a setup composed of hot and cold microbaths. Assume that the hot microbath is an $N$-level system where levels $m$ and $m+1$ are degenerate, $E_{m}^{h}=E_{m+1}^{h}$. The task at hand is to increase the polarization of these two levels, i.e., to increase the population difference $\left|p_{m}^{h}-p_{m+1}^{h}\right|$ by interacting with another microbath. Note that, due to interactions of other levels with the external driving and the other microbath, the total average energy of the hot bath and its entropy can either grow or decrease as the polarization is increased. Consequently, this task is "fine grained," and the quantities appearing in Eq. (10) or (11) do not set explicit bounds on the execution of this task.

Initially, the hot microbath is in a Gibbs state and both levels are equally populated. In the case in which the hot microbath is a two-level system, polarization creation is very similar to the Landauer principle that assigns a minimal heat cost to entropy changes $\Delta S_{h}=-\beta_{c} \Delta\left\langle H_{c}\right\rangle$. The most familiar case is a full resetting of a fully unknown state whose initial entropy is $\ln 2$.

The Landauer erasure principle is one of the central results regarding the energetic consequences of handling information. It highlights the costs that must accompany logically irreversible operations on a subsystem. Thus, it is of interest to study the energetic cost of polarization creation that is the expectation value analog of the Shannon-von Neumann information erasure.

For simplicity, let us first assume that $\beta_{c} \geq \beta_{c}^{\star}$ and relax this assumption later. Moreover, for brevity, we also assume that only two levels are degenerate $\left(E_{m}^{h}=E_{m+1}^{h}=\right.$ $E$ ), and define $E_{+} \equiv E_{m+2}^{h}>E, E_{-} \equiv E_{m-1}^{h}<E$, i.e., the first upper and lower levels around the degeneracy. The population difference of interest can be recast as the expectation value of the operator $A=|m+1\rangle\langle m+1|-| m\rangle\langle m|$, namely $\operatorname{tr}(\rho A)=p_{m+1}^{h}-p_{m}^{h}$. Next, we construct the operator $\widetilde{B}(\xi)=\beta_{c} H_{c}+\beta_{h} H_{h}+\xi \beta_{h} A$ by adding a term proportional to $A$ to the globally passive operator $\mathcal{B}$. As shown in Fig. 9(a), this operator splits the degeneracy.

From the no-crossing rule of the passivity deformation framework, we find that $\widetilde{B}(\xi)$ is globally passive for $-v \leq \xi \leq v$, where $v=\min \left(E_{+}-E, E-E_{-}\right)$. By combining the $-v$ bound for positive changes in $A$ with the $+v$ bound for negative changes we obtain

$$
\beta_{c} \Delta\left\langle H_{c}\right\rangle+\beta_{h} \Delta\left\langle H_{h}\right\rangle \geq v \beta_{h}\left|p_{m+1}^{h}-p_{m}^{h}\right|
$$


(a)

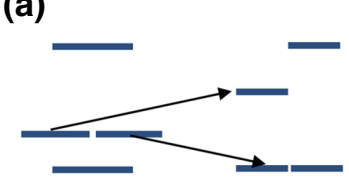

(b)

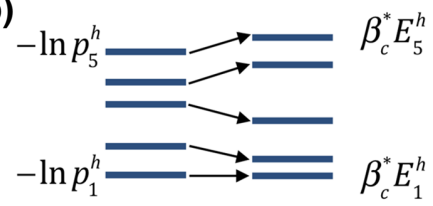

FIG. 9. (a) The deformation for obtaining a bound on the polarization erasure in the nonoverlapping ladders regime (only one ladder is plotted). (b) The deformation that assigns a temperature to a nonthermal passive environment (one ladder is shown - nonoverlapping ladders regime).

where the polarization $p_{m+1}^{h}-p_{m}^{h}$ is calculated at the end of the process (initially there is no polarization). If $\beta_{c}=\beta_{h}=$ $\beta$ or, alternatively, if there is only one microbath with a Hamiltonian $H$, and the goal is to polarize two of its levels, then Eq. (31) becomes

$$
W \geq v\left|p_{m+1}^{h}-p_{m}^{h}\right|
$$

where $W=\Delta\langle H\rangle$ is the work needed to create the polarization. A more restrictive inequality than that in Eq. (31) can be obtained by replacing $\beta_{c}$ with $\beta_{c}^{\star}$, resulting in

$$
\frac{\omega_{h}^{\max }}{\omega_{c}^{\min }} \Delta\left\langle H_{c}\right\rangle+\Delta\left\langle H_{h}\right\rangle \geq v\left|p_{m+1}^{h}-p_{m}^{h}\right| .
$$

When $\beta_{c}<\beta_{c}^{\star}$, a similar inequality to that in Eq. (31) holds. The only difference is in the expression for $E_{ \pm}$. For $\beta_{c}>\beta_{c}^{\star}$, it is clear that the levels in $\widetilde{B}(v)$ that are closest to the degenerate levels $E_{m}^{h}, E_{m+1}^{h}$ in a specific ladder are in the same ladder, since the ladders are well separated. When the ladders overlap, the closest levels may originate from different ladders. Nonetheless, the principle remains the same, and $v$ is obtained from the maximal deformation before the nearest level is crossed. Note that, for $\beta_{c}<\beta_{c}^{\star}$, it is not possible to replace $\beta_{c} \rightarrow \beta_{c}^{\star}$ as done in Eq. (33) for the case where the ladders do not overlap.

We stress that bound (33) is tight for environments of any size (there is always a BSP operation that saturates the bound). In contrast, the Landauer bound is loose when the environment is small (see the explanation in Appendix C). Furthermore, if the dynamics respects some conservation laws that exclude the possibility of executing the bound-saturating operation (Sec. III B), we can incorporate the conservation laws into Eq. (31) or (33) as done in Sec. IV (second box). As a result, a new attainable bound is obtained where the value of the new $v$ is greater than the value of $v$ without the conservation law.

Next, we consider the case where the energy level of both objects (hot and cold) are an integer multiplication of some frequency $\omega$, i.e., $E_{c(h)} \in\{n \omega\}_{n=1,2,3, \ldots}$. This spectrum can correspond to a spin bath of arbitrary number $N$ and/or to a harmonic oscillator. Furthermore, the initial inverse temperature of the objects are equal, $\beta_{c}=\beta_{h}$. Let $m$ and $m+1$ be two degenerate levels in one of the objects. Employing the passivity deformation rules as before we find that the work needed to polarize these two level satisfies

$$
W / \omega \geq \frac{1}{2}\left|p_{m+1}-p_{m}\right| .
$$

The factor of half that is missing in Eq. (32) appears here since the ladders overlap in this case. As a result, the deformation has to be smaller to avoid level crossing. This example shows that the current framework can also be applied to systems with very large Hilbert spaces.

\section{Local and global deviations from an initial local equilibrium}

In the previous sections, the goal was to find new inequalities for initial states of several uncoupled and thermal subsystems, where Eq. (10) holds. We now do the opposite and look for scenarios where the initial conditions are such that Eq. (10) may be violated (e.g., initially correlated microbath). Our goal is to use passivity deformation to obtain bounds that are valid in this regime, yet still have the same form of Eq. (10), albeit involving some effective temperatures.

\section{Initially athermal passive subsystem}

Consider a situation in which a subsystem is prepared in state $\rho_{0}^{\text {pass }}$ that is athermal, yet still passive with respect to its Hamiltonian $H_{s}$, i.e., $p_{n} \leq p_{k}$ if $E_{n}^{s} \geq E_{k}^{s}$ and $\left[-\ln \rho_{0}^{\text {pass }}, H_{s}\right]=0$. This athermal system is then coupled to an ultracold microbath by a process that is described by a mixture of unitaries. The global passivity of $-\ln \rho_{0}^{\text {tot }}$ yields $\Delta\left\langle-\ln \rho_{0}^{\text {pass }}\right\rangle+\beta_{c} \Delta\left\langle H_{c}\right\rangle \geq 0$. Unfortunately, this expression provides a prediction on the observable $-\ln \rho_{0}$ and not on the energy of the initially passive system. Our goal now is to derive an inequality that would constrain the variation of the subsystem energy $\Delta\left\langle H_{s}\right\rangle$.

Since the ladders are now given by the expression $-\ln \rho_{0}^{\text {pass }}$ instead of $\beta_{h} H_{h}$, the nonoverlapping ladders condition now reads

$$
\beta_{c} \geq \bar{\beta}_{c}^{\star} \doteq \frac{1}{\omega_{c}^{\min }} \ln \left(\frac{p_{1}}{p_{N}}\right) .
$$

In what follows we assume that this condition holds. Consequently, it is possible to use the passivity deformation rules and get a new globally passive operator using the deformation $-\ln p_{i} \rightarrow \beta_{s}^{\text {eff }} H_{s}$, as depicted in Fig. 9(b). The value of $\beta_{s}^{\text {eff }}$ is determined by the no-overlap condition $\beta_{s}^{\text {eff }} \omega_{s}^{\max }=\beta_{c} \omega_{c}^{\min }$. The resulting bound is

$$
\frac{1}{\omega_{c}^{\min }} \Delta\left\langle H_{c}\right\rangle+\frac{1}{\omega_{s}^{\max }} \Delta\left\langle H_{s}\right\rangle \geq 0 .
$$

We have demonstrated that such athermal initial states still lead to bounds that restrict energy exchanges. For example, 


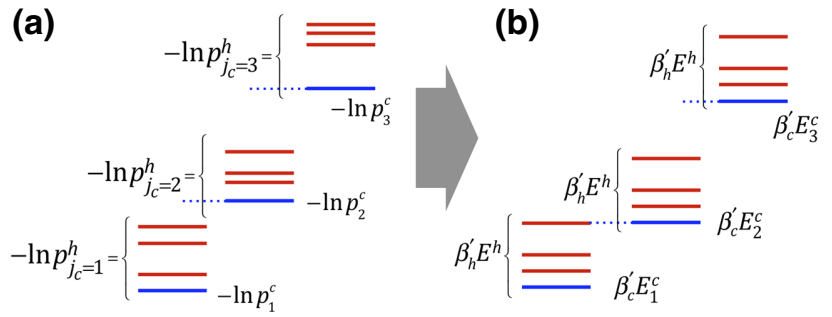

FIG. 10. (a) When two environments are initially classically correlated, the globally passive operator $\mathcal{B}=-\ln \rho_{0}^{\text {pass }}$ no longer has the "ladder replicas" structure shown in Fig. 7. Instead, each ladder is determined by the conditional probability via $-\ln \left(p_{j \mid i}^{h \mid c}\right)$. (b) If the manifolds are separated as shown in (a) then it is possible to deform the original $\mathcal{B}$ into a new operator with a replica structure and thermal local operators that yield a bound of the form $\beta_{c}^{\text {eff }} \Delta\left\langle H_{c}\right\rangle+\beta_{h}^{\text {eff }} \Delta\left\langle H_{h}\right\rangle \geq 0$. This procedure yields an effective temperature related to the initial classical correlation.

in the no-overlap regime an engine exploiting this passive environment is limited by the Otto efficiency with a compression ratio of $\omega_{c}^{\min } / \omega_{s}^{\max }$.

The extension to two athermal small environments in the nonoverlapping ladders regime is straightforward. Denoting the additional athermal environment that replaces the cold microbath by $s^{\prime}$, we find that Eq. (10) remains valid (replacing labels " $c$ " by " $s$ "), but now the no-overlap condition reads

$$
\min \left(\ln p_{n}^{s^{\prime}} / p_{n-1}^{s^{\prime}}\right) \geq \ln p_{1} / p_{N} .
$$

Since Eq. (36) holds in this case as well, the Otto efficiency limits the performance of the engine even though none of the environment is initially thermal. Moreover, exactly as in Sec. IV A2, it is not possible to cool and reduce the average energy of $s^{\prime}$ since the ladders do not overlap.

\section{2. "Inert" classical correlations between subsystems}

Next, we consider a scenario in which there are initial correlations (in the energy basis) between different subsystems. For concreteness, we consider a setup with the initial Hamiltonian $H=H_{c}+H_{h}$. The initial state of the setup is diagonal in the eigenbasis of the Hamiltonian, but does not form a product state, namely $p_{i j}^{c h} \neq p_{i}^{c} p_{j}^{h}$, where the $p$ are the diagonal elements of the density matrix. As before, the setup is driven by a process modeled by a mixture of unitaries. At the end of the process, the coupling is turned off so that the final Hamiltonian is the same as the initial Hamiltonian.

The initial correlations of the type considered here can be achieved by creating interaction between subsystems and turning it off before the process starts. In quantum setups, such a procedure may result in additional quantum correlations. Nevertheless, there are situations where the quantum correlation reduces to classical correlation.
For example, if two objects with different energy gaps are brought momentarily into resonance by a driving field, the free evolution after the drive is switched off will cause the off-diagonal elements of the density matrix to rotate in time. If it is not known when the correlating interaction took place, only the time-averaged density matrix is accessible. As a result, the time-averaged state is classically correlated. Alternatively, if the two objects are subjected to local dephasing after the correlating interaction is switched off, the joint state will be classically correlated.

In the derivation of Eq. (10) the lack of correlations is a crucial assumption (see Ref. [4] and the references therein). Indeed, such initial correlations may result, for example, in spontaneous energy flow from a cold subsystem to a hot subsystem [52]. Below, we show that if one subsystem is sufficiently cold, a second-law-like inequality holds, but with effective temperatures that depend on the initial correlations. We start by writing the classically correlated initial state as

$$
p_{i j}^{c h}=p_{i}^{c} p_{j \mid i}^{h \mid c}
$$

where $p_{j \mid i}^{h \mid c}$ is the conditional distribution of the hot environment given that the cold environment is in state " $i$ ". By taking - ln of the right-hand side of Eq. (38), it becomes clear how to extend the "floors and ladders" diagram to the classically correlated case: $\left\{-\ln \left(p_{i}^{c}\right)\right\}_{i}$ constitute the floors, and they are used for plotting the initial staircase as before. Next, on floor $i$ we put the "conditional ladder" $\left\{-\ln \left(p_{j \mid i}^{h \mid c}\right)\right\}_{j}$ that corresponds to it. That is, the correlation manifests in the fact that the ladders are different from each other as shown in Fig. 10.

In this way, arbitrarily strong classical correlation can be represented. In order to apply passivity deformation and derive a bound that resembles Eq. (10), we make the following assumptions: (1) as in the previous section, the ladders do not overlap; (2) the conditional marginals $\left\{-\ln \left(p_{j \mid i}^{h \mid c}\right)\right\}_{j}$ are passive with respect to $H_{h}$; and (3) $\left\{-\ln \left(p_{i}^{c}\right)\right\}_{i}$ is passive with respect to $H_{c}$. We do not assume that the marginals are thermal or that the correlation is weak.

Starting with the cold subsystem, we first shift the floors from $\left\{-\ln \left(p_{i}^{c}\right)\right\}_{i}$ to $\beta_{c}^{\text {eff }} H_{c}$. Since the ladders do not overlap, there is always some large enough $\beta_{c}^{\text {eff }}$ for which this is possible. To obtain a tight bound, we select the minimal value for which this is possible. Next, to get rid of the correlation in our constructed globally passive operator, we need to make all the ladders identical. Since we assumed that the conditioned marginals of the hot object are passive, it implies that each ladder has the same ordering as $\beta_{h} H_{h}$. Therefore, we can use the ladder separation to deform each ladder separately into $\beta_{h}^{\text {eff }} H_{h}$. For achieving the best bound, $\beta_{h}^{\text {eff }}$ is taken to be the largest value that does not make the ladders cross. 
We conclude that if the three conditions above hold then despite the initial correlations a second-law-like bound of the form

$$
\beta_{c}^{\text {eff }} \Delta\left\langle H_{c}\right\rangle+\beta_{h}^{\text {eff }} \Delta\left\langle H_{h}\right\rangle \geq 0
$$

holds for any mixture of unitaries. The information about the correlations is encapsulated in the values of the effective temperatures. Note that, by construction, this bound is tight (see Sec. III B).

As in Sec. IV A 2, the no-overlap condition leads to $\Delta\left\langle H_{c}\right\rangle \geq 0$. Hence, we call such a correlation an "inert correlation" as it cannot be exploited to cool the cold environment. If, however, the product state created from the product of the marginals $\rho_{\text {prod }}=\operatorname{tr}_{h} \rho_{\text {ch }} \otimes \operatorname{tr}_{c} \rho_{\text {ch }}$ satisfies the no-overlap condition but $\rho_{\text {ch }}$ does not (noninert case), it is possible to cool the object that appears to be locally ultracold.

Several recent papers were devoted to the study of fluctuation theorems of small systems that are strongly coupled to their environment [53-56]. These involve classical and quantum correlations that are typically beyond the scope of the "inert" classical correlation considered here. One should note several important differences between the approach taken in the aforementioned papers and that taken here. Specifically, in the study of fluctuation theorems, the coupling between a system and its environment is always present, so the question of dividing the interaction energy between subsystems becomes nontrivial. In addition, the framework of stochastic thermodynamics deals with fluctuating quantities. Our results pertain to changes of expectation values. Finally, the initial correlation was also treated using observational entropy in Refs. $[9,10]$

\section{COARSE GRAINING AND UNDETECTABLE HEAT LEAKS}

In this part of the paper, we use our framework to obtain two general insights. The first deals with situations where coarse graining takes place. This coarse graining can arise due to limited resolution of the measuring device, or due to the lack of interest in some fine details. The other insight concerns undetectable heat leaks. In both cases, the conclusion is based on passivity deformations that create new degeneracies.

In Appendix D, similar passivity deformations are used for obtaining two more results: the partial Clausius inequality and the binary Clausius inequality. These two inequalities reveal a hierarchical structure that starts with the second law and ends with a majorization condition.

\section{A. Coarse graining}

Consider a situation in which the energy levels in the setup of interest have some internal structure that our detectors cannot resolve. We describe this physical situation by considering a model with two sets of quantum numbers so that the energy eigenstates can be denoted by $|n, m\rangle$. Here $n$ corresponds to the resolvable degrees of freedom, while $m$ refers to the experimentally unresolvable internal structure. Our goal is to write an inequality that depends only on the measurable degrees of freedom $n$.

Let $\rho_{0}^{\text {full }}=\sum_{n=1}^{N} \sum_{m=1}^{M_{n}} p_{n m}|n, m\rangle\langle n, m|$ be the full initial density matrix of the setup. Here $M_{n}$ denotes the number of unresolvable states associated with each value of $n$. Global passivity leads to $\Delta\left\langle\mathcal{B}^{\text {full }}\right\rangle \geq 0$, where $\mathcal{B}^{\text {full }}=$ $-\ln \rho_{0}^{\text {full }}$. This inequality clearly depends on all the degrees of freedom of the setup including the internal ones that we wish to coarse grain. We ask under what conditions it holds that

$$
\Delta\left\langle\mathcal{B}^{\mathrm{CG}}\right\rangle \geq 0
$$

where $\mathcal{B}^{\mathrm{CG}}=\sum_{n=1}^{N} q_{n}|n\rangle\langle n|$ and the $q_{n}$ are some real numbers that we obtain shortly.

It is useful to start by examining a special case where the coarse graining is straightforward. When the probabilities are degenerate in the index $m$, we can write $p_{n m}^{0}=$ $\widetilde{p}_{n}$, where the dimension (number of levels) of $\tilde{p}_{n}$ is $N$, which is smaller than the dimension of the original system $\sum_{n=1}^{N} M_{n}$. Such degeneracies can arise, for example, in thermal states due to degeneracies in the Hamiltonian. These degeneracies enable one to simplify the inequality $\Delta\left\langle\mathcal{B}^{\text {full }}\right\rangle \geq 0$ :

$$
\begin{aligned}
0 & \leq \Delta\left\langle\mathcal{B}^{\text {full }}\right\rangle \\
& =\sum_{n m}\left(p_{n m}^{f}-p_{n m}^{0}\right)\left[-\ln p_{n m}^{0}\right] \\
& =\sum_{n}\left(p_{n}^{f}-p_{n}^{0}\right)\left[-\ln \tilde{p}_{n}\right] \\
& =\Delta\left\langle\mathcal{B}^{\mathrm{CG}}\right\rangle .
\end{aligned}
$$

Here $p_{n}^{f, 0} \equiv \sum_{m} p_{n m}^{f, 0}$ and $\mathcal{B}^{\mathrm{CG}}=-\sum \ln \tilde{p}_{n}|n\rangle\langle n|$.

When the probabilities $p_{n m}^{0}$ are not degenerate in the quantum number $m$, the passivity deformation approach can be used to find out if, and under what conditions, one can obtain a coarse-grained inequality. Consider the case shown in Fig. 11(a) where the values $\mathcal{B}_{n m}=-\ln p_{n m}^{0}$ are clustered (in the vertical axis), with different values of $n$ denoting different clusters, while $m$ differentiates between states in the same cluster. Passivity deformation allows one to deform $\mathcal{B}_{n m}$ into a new passive operator $\widetilde{\mathcal{B}}_{n m}$ that is independent of $m$, as depicted in Fig. 11(b). Then, one can repeat the argument in Eq. (41) and obtain a lower-dimensional operator that satisfies the effective inequality (40). This coarse-graining procedure is allowed as long as the different clusters do not overlap. The result is not unique, since the value of $q_{n}$ can be modified 


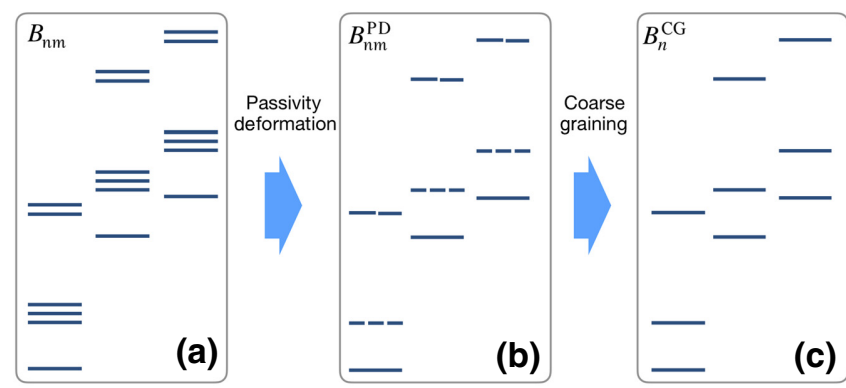

FIG. 11. (a) and (b) The passivity deformation that makes all the unmeasured degrees of freedom degenerate. (b) and (c) After this deformation, it is possible to coarse grain and ignore the internal structure of the levels. This process is possible only when the internal manifolds do not overlap. This is a weaker condition compared to the full no-overlap situation shown in Fig. 7(b).

by deformations. However, there is no point in doing deformations that are below the resolution of the detector.

Note that, in general, the passivity-based inequality $\Delta\left\langle\mathcal{B}^{\mathrm{CG}}\right\rangle \geq 0$ is different from that obtained by first coarse graining the probability distribution and then applying passivity. If the coarse graining is done first, one finds that $B_{n}^{\prime}=-\ln p_{n}^{0}=-\ln \sum_{m} p_{n, m}^{0}=-\ln M_{n} \widetilde{p}_{n}$, where $M_{n}$ is the degeneracy of level $n$. If $M_{n}=M$, i.e., all the $n$ levels have the same degeneracy, then $B_{n}^{\prime}=-\ln \tilde{p}_{n}+$ const, where the additive constant can be ignored as it drops out when calculating $\Delta\left\langle B^{\prime}\right\rangle$. We conclude that, if $M_{n}=M$, it holds that $\mathcal{B}_{n}^{\mathrm{CG}}=B^{\prime}+$ const. That is, in this special case it is not important if the coarse graining is done in the probability space or in the passive operator space. Yet, in general, $\mathcal{B}_{n}^{\mathrm{CG}}$ is not equivalent to $B^{\prime}$. For example, for a thermal state with some degenerate energy structure $E_{n m}=E_{n}$, it holds that $\mathcal{B}_{n}^{\mathrm{CG}}=\beta E_{n}$ while $B_{n}^{\prime}=\beta E_{n}-$ $\log M_{n}$. However, passivity provides an inequality involving $\mathcal{B}_{n}^{\mathrm{CG}}$ and not $B_{n}^{\prime}$. Thus, this example illustrates the importance of coarse graining the passive operator and not the probabilities.

A related, but different approach to obtain a coarsegrained inequality is based on the notion of observational entropy [7-10]. However, the inequality differs from that presented above for two reasons. It involves entropy calculations at the final time. It also does not use the freedom that passivity gives to change the operator whose expectation value is calculated.

\section{B. Undetectable heat leaks}

When studying the dynamics of small quantum systems, we implicitly assume that we can neglect its interactions with some parts of the world. But it is hard to be certain when such assumptions are valid. Perhaps our system is weakly interacting and exchanging energy with some unknown environment. As explained before, it is possible to use violations of inequalities that hold for unitary evolution to detect these potential heat leaks. Assuming that only observables in the energy basis are used, it is important both from a fundamental and a practical point of view to understand the limitations of this approach. Next, we exploit passivity deformation to derive a bound that defines scenarios where this approach fails. An environment is said to be detectable if there is an inequality based on expectation values (in the visible system only) that is violated due to the interaction with the hidden environment. The simplest undetectable heat leak takes place when the system interacts with an environment with infinite temperature. For $T_{\mathrm{env}} \rightarrow \infty$, the hidden environment is in a fully mixed state, so the initial state is

$$
\rho_{0}^{\text {tot }}=\rho_{0}^{\text {sys }} \otimes I_{\text {env }} / N_{\text {env }},
$$

where $I_{\text {env }}$ is the identity operator in the environment subspace and $N_{\text {env }}$ is the number of states. After taking the log we obtain

$$
\mathcal{B}=-\ln \rho_{0}^{\text {sys }} \otimes I_{\text {env }}+\text { const. }
$$

From global passivity, $\Delta\langle\mathcal{B}\rangle \geq 0$ holds for any global unitary that involves the system and the fully mixed environment. However, Eq. (43) implies that, for this specific environment, the globally passive operator $\mathcal{B}=B^{\text {sys }} \otimes I_{\text {env }}$ and, therefore,

$$
\Delta\left\langle B^{\text {sys }}\right\rangle=\Delta\langle\mathcal{B}\rangle \geq 0 .
$$

We conclude that, for this environment, any passive operator in the system subspace is also globally passive in the full space that included the environment. Consequently, there is no passivity inequality (in the system subspace) that can detect the heat leak associated with $T_{\text {env }} \rightarrow \infty$ environments.

We now ask whether this undetectability is unique to $T_{\text {env }} \rightarrow \infty$ environments, or can it appear in finite temperatures as well. An example of a typical operator $\mathcal{B}_{\text {vis }}=$ $-\ln \rho_{\text {vis }}(0)$ is plotted with blue dotted lines in Fig. 12(a). As explained in Sec. IV, with the lack of initial correlations, the operator $\mathcal{B}_{\text {tot }}=-\ln \rho_{\text {vis }}(0)-\ln \rho_{\text {env }}(0)$ can be plotted by using the visible part as the floors [blue lines in Fig. 12(a)] and the environment as ladders (red lines). Without loss of generality, we can set the lowest energy of the environment to be zero. In the example plotted in Fig. 12(a) each ladder is fully contained between the gaps of the visible systems. As such, the ladders do not overlap and it is possible to apply a passivity deformation that brings all the levels in each red ladder to the ground state of the ladder (Fig. 12). The resulting globally passivity operator is $\mathcal{B}_{\text {tot }}=-\ln \rho_{\text {vis }}(0)$ [the term $\ln I_{\text {env }} / \operatorname{dim}($ env $)$ is a constant shift that can be dropped].

By reversing this logic we conclude that, if $T_{\text {env }}$ is sufficiently high ( $\beta_{\text {env }}$ is sufficiently small), any operator that is 
(a)

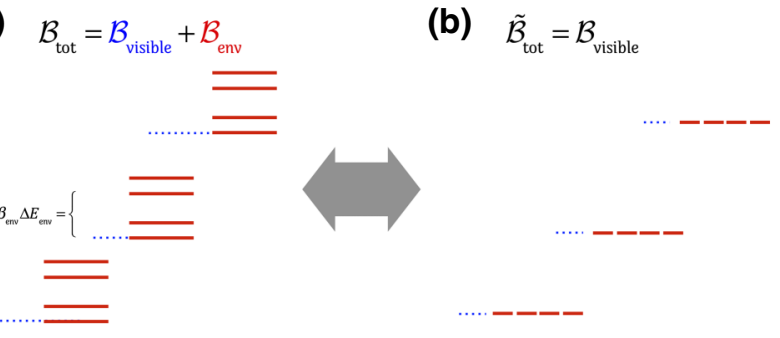

FIG. 12. (a) A representation of a globally passive operator (in the space of the whole setup, i.e., visible + environment) when the initial temperature of the hidden environment is sufficiently hot so that the "ladders" of the environment (red) do not overlap. Such operators can be deformed into the operator shown in (b) according to the rules in Sec. IV. From this we conclude that, whenever the temperature of the environment is sufficiently high [see bound (45)], the ladders do not overlap and this environment is not detectable by any passivity deformation inequality.

globally passive with respect to the visible system is also globally passive with respect to the whole setup (visible + environment). Consequently, local inequalities cannot be violated by a mixture of unitaries on the whole setup. The environment temperature above which heat leaks are undetectable is determined by the no-overlap condition for the environment ladders $\beta_{\text {env }}\left[\max \left(E_{\text {env }}\right)-\min \left(E_{\text {env }}\right)\right] \leq$ $\min \left(\mathcal{B}_{n}^{\text {vis }}-\mathcal{B}_{n-1}^{\text {vis }}\right)$ or

$$
T_{\text {env }} \geq T_{\text {undet }} \doteq \frac{\max \left(E_{\text {env }}\right)-\min \left(E_{\text {env }}\right)}{\min \left(\mathcal{B}_{n}^{\text {vis }}-\mathcal{B}_{n-1}^{\text {vis }}\right)}
$$

In summary, although $T_{\text {undet }}<\infty$ and the associated reduced dynamics is not a unital map, an environment with initial temperature $T_{\text {env }} \geq T_{\text {undet }}$ cannot be detected. The converse is true in the following sense: for $T_{\text {env }} \leq$ $T_{\text {undet }}$, there exists a unitary map for which the environment can be detected using only observables of the visible system.

From a practical point of view, Eq. (45) is useful only if the maximal energy gap of the environment is known and the environment is initially uncorrelated with the system. Yet, the goal here was just to show that $T_{\text {env }} \rightarrow \infty$ is not the only case that leads to undetectable heat leaks.

\section{CONCLUSION}

In this paper, we present a framework that enables us to derive thermodynamiclike inequalities for fine-grained observables that are becoming measurable in various setups such as ion traps, optical lattices, superconducting qubits, and more. These observables do not appear in the second law and are therefore not constrained by it. On top of being applicable to new observables, the passivity deformation framework also overcomes some of the limitations that arise when using the analogue of the second law in microscopic setups: it yields tight bounds in scenarios where other second-law-like inequalities, such as Eqs. (10) and (11), are not tight, it can be integrated with dynamical constraints due to conservation laws, and it produces informative and useful results even when one of the environments is very cold.

What makes all this possible is the exploitation of the energy spectrum of the various elements in the setup. In man-made setups (superconducting circuits, ion traps, etc.) this information is readily available. Thus, we believe that our method is more suitable for man-made setups and less suitable for natural and biological systems. We note that the denser the spectrum of the environment with respect to the system, the less room there is to do a deformation. This can be potentially circumvented by a dynamical constraint or a conservation law that increases the space of possible deformations.

The added value of our inequalities is that they deal with measurable expectation values and they are indifferent to the complexity of the evolution. This feature is desirable in systems that cannot be classically simulated like quantum computers and quantum simulators.

In the future, heat machines may not be restricted to cooling and work extraction. For example, quantum machines can be used to build up entanglement $[5,6]$ or to manipulate some observables that are not directly associated with energy or entropy. The upper bounds on the performance of such machines can be identified using a thermodynamiclike approach, without the need to explicitly solve for the dynamics. In this work, we show the applicability of our framework for setting bounds on the performance of such machines.

We demonstrate that the passivity deformation framework does not only produce bounds but also insights. By exploring the tightness of the bounds, path-independent processes, which may not correspond to reversible processes, are identified. In addition, we identify scenarios where processes with athermal and correlated environments satisfy inequalities that take the same form of as the second law, yet with some effective temperature. Moreover, passivity deformation offers a clear recipe for coarse graining and provides the conditions for the validity of the bounds when some degrees of freedom cannot be resolved in the measurement process.

For future research, it would be interesting to explore the application of the theory to setups with particle transport, band-gapped materials, or steady-state operation. In particular, band-gapped materials offer the freedom to execute deformations when they interact with a system that has a small Hilbert space (e.g., a probe). It is also of interest to investigate to what extent these inequalities can reveal that a system is not well isolated from the rest of the world [3], e.g., due to the presence of heat leaks or lazy Maxwell demons [2]. The detection of heat leaks can be used to compare the predictive power of 
different thermodynamiclike approaches, such as passivity deformation, thermodynamic resource theory, and stochastic thermodynamics [1].

Our findings can be verified in various quantum setups such as ion traps, neutral atoms in optical lattices, or in presently available superconducting quantum processors [57]. A proof-of-principle experimental demonstration of superior heat leak detection based on passivity deformation was successfully carried in out in a companion paper using the IBM quantum processors [1].

\section{ACKNOWLEDGMENTS}

R.U. is grateful for support from the Israel Science Foundation (Grant No. 2556/20). S.R. is grateful for support from the U.S.-Israel Binational Science Foundation (Grant No. 2014405) and from the Israel Science Foundation (Grant No. 1526/15). We thank G. Landi and J. Pereira dos Santos for their assistance in preparing Fig. 8.

\section{APPENDIX A: PASSIVITY AS A BINARY RELATION}

To methodically study the global passivity of various operators with respect to $\rho_{0}$, we introduce in this appendix the ordering function tool. Let $A$ and $B$ ( $B$ is unrelated to $\mathcal{B})$ be two Hermitian matrices of the same dimensionality; the (mutual) ordering function is given by

$$
\chi(A, B) \doteq \operatorname{tr}(A B)-\lambda_{A}^{\downarrow} \cdot \lambda_{B}^{\downarrow}
$$

where $\lambda_{A(B)}^{\downarrow}$ are the eigenvalues of $A(B)$ sorted in decreasing order. The matrices $A$ and $B$ have the same ordering if and only if

$$
\chi(A, B)=0 .
$$

Similarly, $A$ and $B$ have reverse ordering if and only if

$$
\chi^{\downarrow \uparrow}(A, B)=0,
$$

where the reverse ordering function is

$$
\chi^{\downarrow \uparrow}(A, B) \doteq \operatorname{tr}(A B)-\lambda_{A}^{\downarrow} \cdot \lambda_{B}^{\uparrow} .
$$

In this notation, the two conditions for global passivity in Eq. (3) can be jointly written as

$$
\chi^{\downarrow \uparrow}\left(A, \rho_{0}^{\text {tot }}\right)=0 .
$$

This can be used to numerically determine the value of the critical $\xi_{ \pm}$in the operator $\mathcal{B}+\xi A$ [see Eqs. (12)-(14)]. The first step is to plot $\chi^{\downarrow \uparrow}\left(\mathcal{B}+\xi A, \rho_{0}^{\text {tot }}\right)$ as a function of $\xi$. By construction, at $\xi=0$ it holds that $\chi^{\downarrow \uparrow}\left(\mathcal{B}+\xi A, \rho_{0}^{\text {tot }}\right)=0$. Starting from $\xi=0$, the first positive (negative) instance of where $\chi^{\downarrow \uparrow}\left(A, \rho_{0}^{\text {tot }}\right) \neq 0$ will determine the value of $\xi_{+}$ $\left(\xi_{-}\right)$.

The two ordering functions have some useful properties. Let $f_{C}$ be a strictly monotonic decreasing function $f_{C}^{\prime}(x)<0$ in the spectral range of operator $C, x \in$ $\left[\min \left(\lambda_{C}\right), \max \left(\lambda_{C}\right)\right]$, and similarly $g_{C}$ satisfies $g_{C}^{\prime}(x)>0$ in the same regime. Then it holds that

$$
\begin{aligned}
\chi^{\downarrow \uparrow}(A, B)=0 & \Longleftrightarrow \quad \chi\left[f_{A}(A), B\right]=0, \\
& \Longleftrightarrow \chi\left[A, f_{B}(B)\right]=0, \\
\chi(A, B)=0 \quad & \Longleftrightarrow \chi\left[A, g_{B}(B)\right]=0, \\
& \Longleftrightarrow \chi\left[g_{A}(A), B\right]=0 .
\end{aligned}
$$

Using $f_{B}(B)=-\ln B$ in Eq. (A6), we conclude that the global passivity condition (A5) for operator $A$ can be written as

$$
\chi(A, \mathcal{B})=0 \quad(\text { global passivity of } A),
$$

where $\mathcal{B}=-\ln \rho_{0}^{\text {tot }}$ as before Eq. (8). As a reassurance exercise, we set $A=\mathcal{B}$ and find that $\mathcal{B}$ is globally passive, since any operator is ordered with respect to itself. Next, we use property (A7) to deduce that

$$
\chi\left[g_{\mathcal{B}}(\mathcal{B}), \mathcal{B}\right]=0
$$

from $\chi(\mathcal{B}, \mathcal{B})=0$. Choosing $g_{\mathcal{B}}(x)=\operatorname{sgn}(\alpha) x^{\alpha}$ we get the global passivity inequalities

$$
\Delta\left\langle\operatorname{sgn}(\alpha) \mathcal{B}^{\alpha}\right\rangle \geq 0
$$

for any evolution of the form (5) in the setup [2].

Ordering as defined in Eq. (A2) can be viewed as a binary relation: $A \sim B \Leftrightarrow \chi(A, B)=0$. While this binary relation is reflexive, $A \sim A$, and symmetric, $A \sim B=B \sim$ $A$, it may not be transitive, i.e., it is possible that $A \sim B$ and $B \sim C$ but $A \nsim C$. Hence, in general, $\chi=0$ is not an equivalence relation.

The breakdown of transitivity takes place if $A$ and $B$ have different degeneracy structures. We say that $B$ is nonequivalent to $A$ if (1) $A \sim B$ and (2) at least two eigenvectors that are nondegenerate in $A$ are degenerate in $B$, i.e., there are at least two eigenvectors $v_{k}, v_{l}$ such that

$$
\left\langle v_{k}|A| v_{k}\right\rangle \neq\left\langle v_{l}|A| v_{l}\right\rangle,
$$

$$
\left\langle v_{k}|B| v_{k}\right\rangle=\left\langle v_{l}|B| v_{l}\right\rangle .
$$

Now, it is possible to construct an operator $C$ that satisfies $B \sim C$ but not $A \sim C$. For simplicity, for the levels $j \neq k, l$, we choose $C$ that satisfies $\left\langle v_{j}|C| v_{j}\right\rangle=\left\langle v_{j}|A| v_{j}\right\rangle$ that makes all these levels irrelevant, and it is possible to restrict the discussion to $j$ and $l$. Next, we choose the eigenvalues $k$ 
and $l$ in $C$ to have the opposite ordering with respect to $A$. This can be done by the choice $\left\langle v_{l}|C| v_{l}\right\rangle=\left\langle v_{k}|A| v_{k}\right\rangle$ and $\left\langle v_{k}|C| v_{k}\right\rangle=\left\langle v_{l}|A| v_{l}\right\rangle$. By construction, $A \nsim C$ since the ordering is opposite in $k$ and $l$. Yet, due to the degeneracy in $B$, it holds that $A \sim B$ and $B \sim C$.

This example explains why in the deformation rules in Sec. IV we can only split degeneracies that were already there in the initial density matrix. Otherwise, it would contradict the noncrossing rule: we can first make the two states degenerate and then split them in the other direction, which creates a forbidden crossing. Finally, we point out the relation between ergotropy and the ordering function. Even when $A$ and $B$ are some local Hamiltonians, the ordering function does not depend on the energy eigenvalues (it is not even an observable) and it does not impose any limitation on the evolution. It is simply a relation between two matrices. However, the passive state that determines the ergotropy satisfies $\chi^{\downarrow \uparrow}\left(\rho_{\text {pass }}, H\right)=0$. In the context of passivity-based inequalities, we require that the evolution is a mixture of unitaries, and that the initial state has a reverse ordering with respect to $\rho_{0}^{\text {tot }}$.

\section{APPENDIX B: OPTIMAL PROTOCOLS FROM PASSIVITY}

Consider a setup that aims to achieve a maximal change in the expectation value of a certain observable of interest A (a Hermitian operator). The observable may be "local," i.e., involve only one element of the setup or it may be global and involve several elements or even the whole setup. For example, in refrigerators, the goal is to minimize the average energy of a cold subsystem $\langle A\rangle=\left\langle H_{c}\right\rangle$, which is a local quantity. In engines, the goal is to reduce the energy of the whole setup $\langle A\rangle=\left\langle H_{\text {tot }}\right\rangle$ (global quantity), since this change is equal to the amount of work exchanged with the driving field that executes the protocol. Note, however, that $A$ does not have to be related to energy or to the original basis of the initial state of the setup. Here $A$ can be any Hermitian operator bounded from below in the Hilbert space of the setup (see, e.g., the dephasing example in Sec. III C 2).

Let us assume that the initial state of the setup $\rho_{0}^{\text {tot }}$ is given, and so is the operator $A$ that describes the observable of interest. Thus, the initial expectation value of $A$, $\langle A\rangle_{0}=\operatorname{tr}\left[\rho_{0}^{\text {tot }} A\right]$, is fixed. Our goal is to find the optimal unitary $U_{\text {opt }}$ that will produce the lowest value of $\langle A\rangle$, i.e., $A_{0} \rightarrow A_{\text {min }}=\operatorname{tr}\left[\rho_{\text {opt }}^{\text {tot }} A\right]=\operatorname{tr}\left[U_{\text {opt }} \rho_{0}^{\text {tot }} U_{\text {opt }}^{\dagger} A\right]$. Fortunately, this problem is already solved by the principle of passivity. Adopting the logic of passivity, finding $U_{\text {opt }}$ is simple, the unitary that transforms $\rho_{0}^{\text {tot }}$ into a passive state with respect to $A: \chi^{\downarrow \uparrow}\left(\rho_{\mathrm{opt}}^{\text {tot }}, A\right)=0$ will do the job. This can be carried out in two steps. The first step is to rotate $\rho_{0}^{\text {tot }}$ to the basis of $A$ (if it is not already in this basis). The second step is to apply simple level permutations that will rearrange the populations in a monotonically decreasing order with respect to the eigenvalue of $A$.

If the operator is local as in the case a refrigerator $A=H_{c}$, it is important to write it in the Hilbert space of the whole setup $H_{c} \rightarrow H_{c} \otimes I_{\text {rest }}$, where $I_{\text {rest }}$ is the identity operator of the rest of the setup. As an example, consider the case of a qutrit with energy spacings $\omega$ that is being cooled by two spins with energy spacing $\omega$. All the particles start at thermal equilibrium with inverse temperature $\beta$. The optimal protocol is obtained by building a bar plot (see Fig. 13) where the $x$ axis contains the sorted eigenvalues of $A$. Local operators such as $H_{c}$ exhibit many degeneracies, but their ordering with respect to each other makes no difference in finding the minimal value of $\langle A\rangle$. The $y$ axis in Fig. 13 is the probability of populating each eigenstate of $H_{c} \otimes I_{\text {rest }}$ according to the initial distribution determined by $\rho_{0}^{\text {tot }}$. If the distribution is monotonically decreasing, it implies that $\rho_{0}^{\text {tot }}$ and $H_{c} \otimes I_{\text {rest }}$ are passive with respect to each other and $\langle A\rangle$ is already in its minimal
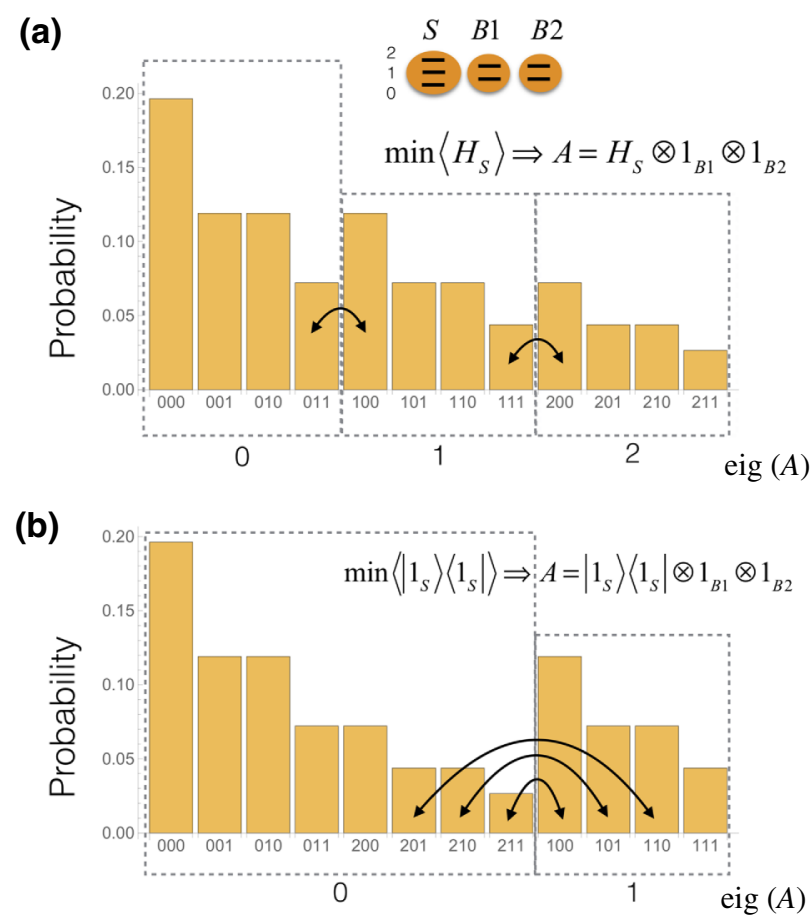

FIG. 13. Optimal protocols for manipulating a qutrit system $(S)$ using two qubits ( $B 1$ and $B 2$ ). (a) To reduce the average energy of $S$, the probability distribution of the eigenvalues of $A=$ $H_{S} \otimes I_{B 1} \otimes I_{B 2}$ are plotted. The optimal protocol (black arrows) is obtained by applying the permutations that lead to a monotonically decreasing distribution of $A$. (b) Optimal protocol for an $X$ machine (see the text). Here the task is to maximally deplete level number 1 of the system. For this, we set $A=\left|1_{S}\right\rangle\left\langle 1_{S}\right| \otimes I_{B 1} \otimes I_{B 2}$ and redo the plot. Note that the permutation inside each eigenvalue block (dashed rectangular) has no impact on the final value of $\langle A\rangle$. Thus, a smaller number of permutations (black arrows) can be used compared to the full sorting of the probability distribution. 
passive value. However, if the distribution is nonmonotonically decreasing as in Fig. 13(a), it is clear that the needed unitary is the one that rearranges the distribution into a monotonically decreasing form.

As a second example, we consider an exotic heat machine whose goal is to deplete the population of the middle level ("1") of the qutrit. We use the setup shown in Fig. 13. This time the operator of interest is $A=\left|1_{S}\right\rangle\left\langle 1_{S}\right| \otimes$ $I_{B 1} \otimes I_{B 2}$. Eigenvalue 1 (respectively 0 ) stands for all the global states of the setup in which the middle level of the system is (respectively is not) populated. As before, we plot the distribution of $A$ [see Fig. 13(b)], and apply sorting permutations to minimize the expectation value of $A$.

Although a complete sorting of the distribution always provides the optimal protocol, it may contain many operations that do not affect the observable of interest $A$. Any operation between degenerate states of $A$ has no impact on $\langle A\rangle$ [i.e., permutation between states in the same dashed box in Fig. 13(b)]. Thus, in some cases, as in this example, a partial sorting can lead to the same optimal performance (same change in $\Delta\langle A\rangle$ ) as shown by the black arrows in Fig. 13(b). In general, the partial sorting protocol differs from the full sorting protocol in the final state of the environment (the two qubits) and its final system-environment correlation.

\section{APPENDIX C: UNATTAINABILITY OF EQ. (11) FOR SMALL ENVIRONMENTS}

As described in Ref. [4] and the references therein (e.g., Ref. [35]), by assuming that the environment is initially in a Gibbs state $\rho_{0}^{\text {env }}=e^{-\beta H^{\text {env }}} / Z$, the following equality holds:

$$
\Delta S^{\mathrm{sys}}+\beta \Delta\left\langle H^{\mathrm{env}}\right\rangle=D\left(\rho_{f} \mid \rho_{f}^{\mathrm{sys}} \otimes \rho_{f}^{\mathrm{env}}\right)+D\left(\rho_{f}^{\mathrm{env}} \mid \rho_{0}^{\mathrm{env}}\right) .
$$

Since the quantum relative entropy satisfies

$$
D(x, y)>0 \quad \text { for } x \neq y,
$$

we find that, if $\rho_{f} \neq \rho_{f}^{\text {sys }} \otimes \rho_{f}^{\text {env }}$ (there is some correlation buildup) or if $\rho_{f}^{\text {env }} \neq \rho_{0}^{\text {env }}$ (the environment is changed by the interaction with the system), then

$$
\Delta S^{\mathrm{sys}}+\beta \Delta\left\langle H^{\mathrm{env}}\right\rangle>0,
$$

and therefore inequality (11) cannot be saturated. In the microscopic weak-coupling limit these two relative entropy terms become negligible. However, in small setups, the environment is often driven far away from equilibrium and non-Markovian dynamics takes place. As a result, these terms can be quite large and bound (C3) is far from being tight.

\section{APPENDIX D: THE TRUNCATED AND BINARY INEQUALITIES}

\section{Truncated inequalities}

In the following, we show a generic prescription (i.e., one that can be carried out in any setup) for constructing inequalities that depend only on parts of the Hilbert space, while ignoring others. These inequalities can exhibit superior heat leak detection or lazy demon detection.

Let us consider a setup with several initial uncorrelated microbaths. We start by considering the operator $\mathcal{B}=-\ln \rho_{0}$, which is globally passive by construction [Fig. 14(a)]. Denoting by $0 \leq b_{1} \leq b_{2} \leq b_{3} \leq \cdots$ the eigenvalues of $\mathcal{B}$ ("levels" of $\mathcal{B}$ ), let us apply the following deformation. First, the lowest level $b_{1}$ is lowered to zero. Since the eigenvalues of $b_{i} \geq 0$, this means moving the level to a point that is lower than all the other levels. Then, the second-lowest level $b_{2}$ is lowered to zero, and this is repeated until only the $l$ highest levels (i.e., the least-populated levels) remain, as shown in Fig. 14(b). According to passivity deformation, the resulting operator $\mathcal{B}^{(l)}=\sum_{k=N-l+1}^{N} b_{i}\left|b_{i}\right\rangle\left\langle b_{i}\right|$ satisfies

$$
\Delta\left\langle\mathcal{B}^{(l)}\right\rangle \geq 0 .
$$

The physical interpretation of such an observable is not self-evident. For example, $\mathcal{B}$ of an initially uncorrelated microbath can be written as a sum of local operators $A_{c} \otimes I_{h}+I_{c} \otimes A_{h}$, but $\mathcal{B}^{(l)}$ in general cannot be written in this form since the remaining "ladders" are not identical. Nevertheless, for a setup composed of several microbaths, the $\mathcal{B}^{(l)}$ are observables in the energy basis, and as such, they can be obtained from energy measurements in the different subsystems.

We have numerically verified that in a linear spin chain where a lazy demon operates between the two middle spins, the $\mathcal{B}^{(l)}$ inequalities can detect demons that $\operatorname{sgn}(\alpha) B^{\alpha}$ cannot detect. This was checked for chains of up to ten spins. At first, it may seem surprising that cropping a few levels from $\mathcal{B}$ can improve the sensitivity. However, while some levels of $\mathcal{B}$ are more affected by feedback or heat leaks, some are not affected, and contribute a positive value to $\Delta\langle\mathcal{B}\rangle \geq 0$. This positive contribution can degrade the detection performance. By excluding these

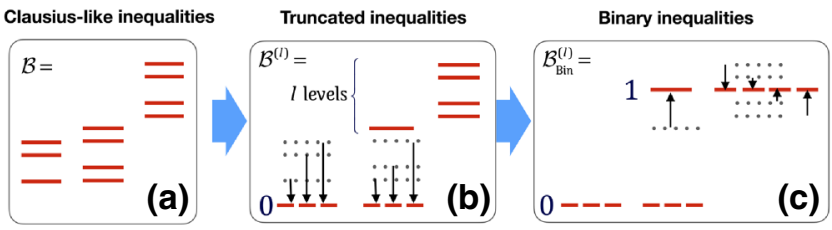

FIG. 14. (a) and (b) The deformation that leads to the truncated inequalities. (b) and (c) The deformation that leads to binary inequalities. 
levels (within the limitations of the passivity deformation rules), better sensitivity may be achieved (depending on the specific demon mechanism).

\section{Binary inequalities and their relation to majorization}

Next, we study a similar deformation and relate it to majorization. By applying the same logic as in the previous deformation, the lowest $N-l+1$ levels can be shifted to 0 as before and the highest $l$ levels to the value of 1 [Fig. 14(c)]. The resulting passivity deformation inequalities are

$$
\Delta\left\langle\mathcal{B}_{\text {Bin }}^{(l)}\right\rangle \geq 0
$$

where $\mathcal{B}_{\mathrm{Bin}}^{(l)}=\sum_{k=N-l+1}^{N}\left|b_{i}\right\rangle\left\langle b_{i}\right|$ are projection operators. These observables are binary. They only test if our system is in a certain subspace of the Hilbert space. At first, these binary inequalities look even stranger than Eq. (D1), but they can be understood directly from the following majorization relation between the initial and final populations in the basis of $\mathcal{B}$. By construction, the initial density matrix is diagonal in the basis of $\mathcal{B}$. Thus, for any evolution (5), the final populations in this basis $\left\{p_{i}^{f}\right\}$ are related to initial ones $\left\{p_{i}^{0}\right\}$ (i.e., the eigenvalues) through the majorization relation (Schur lemma [58])

$$
\sum_{j=1}^{l} p_{\uparrow, j}^{0} \leq \sum_{j=1}^{l} p_{\uparrow, j}^{f} \quad \text { for any } 1 \leq l \leq N,
$$

where the up arrow stands for increasing order. First, we note that

$$
\sum_{j=1}^{l} p_{\uparrow, j}^{0}=\operatorname{tr}\left[\rho_{0}^{\text {tot }} \mathcal{B}_{\text {Bin }}^{(l)}\right] \triangleq\left\langle\mathcal{B}_{\text {Bin }}^{(l)}\right\rangle_{0}
$$

and also that

$$
\sum_{j=1}^{l} p_{\uparrow, j}^{f} \leq \sum_{j=1}^{l} p_{j}^{f}=\left\langle\mathcal{B}_{\mathrm{Bin}}^{(l)}\right\rangle_{f} .
$$

Consequently, from Eqs. (D3)-(D5), it follows that

$$
\begin{aligned}
\left\langle\mathcal{B}_{\mathrm{Bin}}^{(l)}\right\rangle_{0} & =\sum_{j=1}^{l} p_{\uparrow, j}^{0} \\
& \leq \sum_{j=1}^{l} p_{\uparrow, j}^{f} \\
& \leq\left\langle\mathcal{B}_{\mathrm{Bin}}^{(l)}\right\rangle_{f} \quad \text { for any } 1 \leq l \leq N,
\end{aligned}
$$

which yields Eq. (D2). Conditions (D2) are identical to majorization (D3) when the final populations have the same ordering as the initial population (and then $\left.\sum_{j=1}^{l} p_{\uparrow, j}^{f}=\left\langle\mathcal{B}_{\text {Bin }}^{(l)}\right\rangle_{f}\right)$.

Since any $\Delta\left\langle\mathcal{B}^{(l)}\right\rangle \geq 0$, Eq. (D1) can be written as a convex sum of several $\Delta\left\langle\mathcal{B}_{\text {Bin }}^{(l)}\right\rangle \geq 0$ inequalities; it follows that any violation of Eq. (D1) is associated with a violation of at least one of the inequalities in Eq. (D2), but not necessarily the other way around. The family of inequalities in Eq. (D2) is more restrictive than Eq. (D1). However, the inequalities in Eq. (D2) do not carry information on the changes in the energies of the subsystems.

Finally, we point out that inequalities (10), (D1), and (D2) and majorization (in this order) form a hierarchal structure that reflects a trade-off between physical context [maximal for Eq. (10)] and tightness (maximal for majorization).

[1] Raam Uzdin and Nadav Katz, Experimental detection of microscopic environments using thermodynamic observables, arXiv:1908.08968 (2019).

[2] Raam Uzdin and Saar Rahav, Global Passivity in Microscopic Thermodynamics, Phys. Rev. X 8, 021064 (2018).

[3] Bartłomiej Gardas and Sebastian Deffner, Quantum fluctuation theorem for error diagnostics in quantum annealers, Sci. Rep. 8, 17191 (2018).

[4] Raam Uzdin, in Thermodynamics in the Quantum Regime - Recent Progress and Outlook, edited by F. Binder, L. A. Correa, C. Gogolin, J. Anders, G. Adesso, (Springer International Publishing, 2018).

[5] Shishir Khandelwal, Nicolas Palazzo, Nicolas Brunner, and Géraldine Haack, Critical heat current for operating an entanglement engine, New J. Phys. 22, 073039 (2020).

[6] Jonatan Bohr Brask, Géraldine Haack, Nicolas Brunner, and Marcus Huber, Autonomous quantum thermal machine for generating steady-state entanglement, New J. Phys. 17, 113029 (2015).

[7] Dominik Šafránek, Joshua M. Deutsch, and Anthony Aguirre, Quantum coarse-grained entropy and thermodynamics, Phys. Rev. A 99, 010101 (2019).

[8] Dominik Šafránek, J. M. Deutsch, and Anthony Aguirre, Quantum coarse-grained entropy and thermalization in closed systems, Phys. Rev. A 99, 012103 (2019).

[9] Philipp Strasberg: Entropy production as change in observational entropy, arXiv:1906.09933 (2019)

[10] Philipp Strasberg and Andreas Winter: Heat, work and entropy production in open quantum systems: A microscopic approach based on observational entropy, arXiv:2002.08817 (2020)

[11] Jader P. Santos, Gabriel T. Landi, and Mauro Paternostro, Wigner Entropy Production Rate, Phys. Rev. Lett. 118, 220601 (2017).

[12] M. Andre, M. Timpanaro, P. Jader, P. Santos, and Gabriel T. Landi, Landauer's principle at zero temperature, arXiv:1911.00910 (2019)

[13] Bruno O. Goes, E. Carlos, and E. Fiore, Landauer's Principle at Zero Temperature, Phys. Rev. Lett. 124, 240601 (2020). 
[14] Udo Seifert, Quantum features of entropy production in driven-dissipative transitions, Phys. Rev. Research 2, 013136 (2020).

[15] Ken Sekimoto, Stochastic Energetics (Springer, 2010), Vol. 799.

[16] John Goold, Marcus Huber, Arnau Riera, Lídia del Rio, and Paul Skrzypczyk, The role of quantum information in thermodynamics - a topical review, J. Phys. A: Math. Theor. 49, 143001 (2016).

[17] Sai Vinjanampathy and Janet Anders, Quantum thermodynamics, Contemp. Phys. 57, 545 (2016).

[18] Giuliano Benenti, Giulio Casati, Keiji Saito, and Robert S. Whitney, Fundamental aspects of steady-state conversion of heat to work at the nanoscale, Phys. Rep. 694, 1 (2017).

[19] Zeeya Merali, The new thermodynamics: How quantum physics is bending the rules, Nat. News 551, 20 (2017).

[20] W. Pusz and S. L. Wornwicz, Passive states and kms states for general quantum systems, Commun. Math. Phys. 58, 273 (1978).

[21] A. Lenard, Thermodynamical proof of the gibbs formula for elementary quantum systems, J. Stat. Phys. 19, 575 (1978).

[22] I. M. Bassett, Alternative derivation of the classical second law of thermodynamics, Phys. Rev. A 18, 2356 (1978).

[23] A. E. Allahverdyan, R. Balian, and Th. M. Nieuwenhuizen, Maximal work extraction from finite quantum systems, Euro. Phys. Lett. 67, 565 (2004).

[24] M. Perarnau-Llobet, K. V. Hovhannisyan, M. Huber, P. Skrzypczyk, N. Brunner, and A. Acín, Extractable Work from Correlations, Phys. Rev. X 5, 041011 (2015).

[25] Wolfgang Niedenzu, Victor Mukherjee, Arnab Ghosh, Abraham G. Kofman, and Gershon Kurizki, Universal thermodynamic limit of quantum engine efficiency, arXiv:1703.02911 (2017).

[26] Mark T. Mitchison, Quantum thermal absorption machines: Refrigerators, engines and clocks, Contemp. Phys. 60, 164 (2019).

[27] Amikam Levy and Ronnie Kosloff, Quantum Absorption Refrigerator, Phys. Rev. Lett. 108, 070604 (2012).

[28] Gleb Maslennikov, Shiqian Ding, Roland Hablützel, Jaren Gan, Alexandre Roulet, Stefan Nimmrichter, Jibo Dai, Valerio Scarani, and Dzmitry Matsukevich, Quantum absorption refrigerator with trapped ions, Nat. Commun. 10, 202 (2019).

[29] Mark T. Mitchison, Mischa P. Woods, Javier Prior, and Marcus Huber, Coherence-assisted single-shot cooling by quantum absorption refrigerators, New J. Phys. 17, 115013 (2015).

[30] Luis A. Correa, José P. Palao, Daniel Alonso, and Gerardo Adesso, Quantum-enhanced absorption refrigerators, Sci. Rep. 4, 3949 (2014).

[31] Luis A. Correa, José P. Palao, Gerardo Adesso, and Daniel Alonso, Performance bound for quantum absorption refrigerators, Phys. Rev. E 87, 042131 (2013).

[32] Mark T. Mitchison, Marcus Huber, Javier Prior, Mischa P. Woods, and Martin B. Plenio, Realising a quantum absorption refrigerator with an atom-cavity system, Quantum Sci. Technol. 1, 015001 (2016).

[33] C. Lindblad, Non-Equilibrium Entropy and Irreversibility, Mathematical Physics Studies Vol. 5 (Springer, 1983).

[34] Asher Peres, Quantum Theory: Concepts and Methods (Springer Science \& Business Media, 2006), Vol. 57.
[35] Massimiliano Esposito, Katja Lindenberg, and Christian Van den Broeck, Entropy production as correlation between system and reservoir, New J. Phys. 12, 013013 (2010).

[36] Ivan Henao and Raam Uzdin, Catalytic transformations with finite-size environments: Applications to cooling and thermometry, arXiv:2010.09070 (2020).

[37] Wolfgang Niedenzu, Victor Mukherjee, Arnab Ghosh, Abraham G. Kofman, and Gershon Kurizki, Quantum engine efficiency bound beyond the second law of thermodynamics, Nat. Commun. 9, 165 (2018).

[38] Takahiro Sagawa, Second law-like inequalities with quantum relative entropy: An introduction, Lect. Quantum Comput. Thermodyn. Stat. Phys. 8, 127 (2012).

[39] Matteo Lostaglio, An introductory review of the resource theory approach to thermodynamics, Rep. Prog. Phys. 82, 114001 (2019).

[40] Fernando Brandão, Michał Horodecki, Nelly Ng, Jonathan Oppenheim, and Stephanie Wehner, The second laws of quantum thermodynamics, Proc. Natl. Acad. Sci. 112, 3275 (2015).

[41] Michał Horodecki and Jonathan Oppenheim, Fundamental limitations for quantum and nanoscale thermodynamics, Nat. Commun. 4, 2059 (2013).

[42] Gilad Gour, Markus P. Müller, Varun Narasimhachar, Robert W. Spekkens, and Nicole Yunger Halpern, The resource theory of informational nonequilibrium in thermodynamics, Phys. Rep. 583, 1 (2015).

[43] Matteo Lostaglio, David Jennings, and Terry Rudolph, Description of quantum coherence in thermodynamic processes requires constraints beyond free energy, Nat. Commun. 6, 6383 (2015).

[44] Krzysztof Ptaszyński and Massimiliano Esposito, Entropy Production in Open Systems: The Predominant Role of Intraenvironment Correlations, Phys. Rev. Lett. 123, 200603 (2019).

[45] Henrik Wilming, Rodrigo Gallego, and Jens Eisert, Second law of thermodynamics under control restrictions, Phys. Rev. E 93, 042126 (2016).

[46] Artemy Kolchinsky and David H. Wolpert, Entropy production and thermodynamics of information under protocol constraints, arXiv:2008.10764, 2020.

[47] Eitan Geva and Ronnie Kosloff, the quantum heat engine and heat pump: An irreversible thermodynamic analysis of The three-level amplifier, J. Chem. Phys. 104, 7681 (1996).

[48] M. J. Henrich, F. Rempp, and G. Mahler, Quantum thermodynamic otto machines: A spin-system approach, Eur. Phys. J. 151, 157 (2007).

[49] Raam Uzdin and Ronnie Kosloff, The multilevel fourstroke swap engine and its environment, New J. Phys. 16, 095003 (2014).

[50] André M. Timpanaro, Jader P. Santos, and Gabriel T. Landi, Landauer's Principle at Zero Temperature, Phys. Rev. Lett. 124, 240601 (2020).

[51] Philipp Strasberg, María García Díaz, and Andreu RieraCampeny, Resurrecting clausius: Why nanomachines are more efficient than we thought, arXiv:2012.03262 (2020).

[52] David Jennings and Terry Rudolph, Entanglement and the thermodynamic arrow of time, Phys. Rev. E 81, 061130 (2010). 
[53] Udo Seifert, First and Second Law of Thermodynamics at Strong Coupling, Phys. Rev. Lett. 116, 020601 (2016).

[54] H. J. D. Miller and J. Anders, Entropy production and time asymmetry in the presence of strong interactions, Phys. Rev. E 95, 062123 (2017).

[55] Christopher Jarzynski, Stochastic and Macroscopic Thermodynamics of Strongly Coupled Systems, Phys. Rev. X 7, 011008 (2017).
[56] Philipp Strasberg and Massimiliano Esposito, Stochastic thermodynamics in the strong coupling regime: An unambiguous approach based on coarse graining, Phys. Rev. E 95, 062101 (2017).

[57] https://www.research.ibm.com/ibmq/technology/experi ence/.

[58] Albert W. Marshall, Ingram Olkin, and Barry C. Arnold, Inequalities: Theory of Majorization and its Applications (Springer, 1979), Vol. 143. 